\title{
Husserl et l'idéalisme classique
}

Rudolf Boehm

\section{Citer ce document / Cite this document :}

Boehm Rudolf. Husserl et l'idéalisme classique. In: Revue Philosophique de Louvain. Troisième série, tome 57, n55, 1959. pp. 351-396;

doi : https://doi.org/10.3406/phlou.1959.5005

https://www.persee.fr/doc/phlou_0035-3841_1959_num_57_55_5005

Fichier pdf généré le 24/04/2018 


\section{Husserl et l'idéalisme classique}

Le propos de la présente étude est d'éclaircir l'évolution des rapports de la pensée de Husserl à la philosophie de l'idéalisme allemand classique (1). Comme point de départ, nous utiliserons quelques remarques autobiographiques de Husserl, qui datent d'une époque où cette évolution touche à sa fin et qui expriment donc une prise de position virtuellement définitive.

En 1924, Husserl, remaniant le texte d'un discours qu'il a fait, le $1^{\text {or }}$ mai de cette même année, à l'Université de Fribourg-enBrisgau, pour célébrer le deuxième centenaire de la naissance de Kant, rédige une étude sur Kant und die Idee der Transzendentalphilosophie ${ }^{(2)}$. Elle est précédée d'une préface où Husserl rappelle que, dès sa phase primitive, le courant phénoménologique manifestait une "forte opposition " à l'égard des "modes de travail " propres à Kant et aux successeurs de celui-ci et qu'il désapprouvait toute tentative en vue de provoquer une " renaissance kantienne " ${ }^{(3)}$. Cependant, continue Husserl, une "parenté essentielle " entre la phénoménologie et la philosophie transcendantale de Kant devait apparaître de façon évidente à l'époque postérieure à la publication des Logische Untersuchungen (1900-1901) et avant même la parution des Ideen zu einer reinen Phänomenologie und phänomenologischen Philosophie (1913) (s).

(1) Nous développons ici, sous forme d'étude historique, quelques-unes des idées que nous avons exprimées dans trois conférences, faites en 1955. à l'invitation de la Faculté de Philosophie de l'Université de Barcelone, sur Le problème de la réduction phénoménologique chez Husserl.

(2) Cf. Edmund HusserL, Gesammelte Werke, publiés par les ArchivesHusserl sous la direction de $H$. L. VAN BREDA, chez Martinus Nijhoff, La Haye, t. VII, pp. 230-287. Dans la suite, nous renvoyons à cette édition en indiquant volume et page.

(8) T. VII, p. 234

(4) T. VII, p. 230 
Le jeune Husserl eut pour maître en philosophie le penseur autrichien Franz Brentano. En 1919, il consigne ses Erinnerungen an Franz Brentano, destinées à être publiées dans un volume commémoratif ${ }^{(5)}$. Il y consacre un passage relativement long à une discussion critique de l'opinion que se faisait Brentano des philosophes de l'idéalisme allemand, opinion que ce dernier cherchait à inculquer à ses disciples. Brentano, rapporte Husserl, " entièrement dévoué à l'idéal austère de la science philosophique la plus rigoureuse (idéal représenté, dans son esprit, par les sciences exactes de la nature), ne voyait dans les systèmes de l'idéalisme allemand qu'une décadence. Etant moi-même, à l'époque de mes débuts, entièrement sous l'influence de Brentano, je n'acquis que bien plus tard cette conviction que partagent à présent beaucoup de chercheurs, pourtant préoccupés d'une philosophie rigoureusement scientifique: à savoir que les systèmes idéalistes... méritent notre plus haute estime " ${ }^{(6)}$.

Si nous cherchons à établir, pour commencer, d'une façon précise les dates de l'évolution husserlienne auxquelles les textes que nous venons de citer font allusion, nous constaterons d'abord que déjà dans les Prolegomena zu einer reinen Logik (1900) qui constituent le premier volume des Logische Untersuchungen, Husserl qualifie d' "idéalistes" ses "intentions en logique " (7) et désigne pareillement $d$ " idéalistes " les arguments qu'il emploie contre les préjugés du "psychologisme " ${ }^{(8)}$. En outre, il s'y réclame de l" " autorité de grands penseurs du passé " et nomme parmi ces derniers, expressément et en premier lieu, Kant ${ }^{(9)}$. Il semble donc que dès 1900. Husserl s'est libéré des préjugés anti-idéalistes que lui avait inspirés son maître Brentano. Il faut du reste tenir compte du fait que les Prolegomena aux Logische Untersuchungen présentent sim-

(s) Oskar Kraus, Franz Brentano, München, 1919. pp. 151-167.

(6) L. c., p. 159. Toutes nos citations de textes allemands seront faites en traduction française. Nous mentionnerons les traductions françaises d'œuvres husserliennes qui existent et dont nous avons pu profiter; cependant, nous avons souvent été obligé de corriger ou de modifier ces traductions dans nos citations.

(7) E. HuSSERL, Logische Untersuchungen, 1900-1901, 1, p. 79, n. 1; traduction française par Hubert ELIE: E. HussERL, Recherches logiques, 1, 1959, p. 85, note 1.

(s) L. c., p. 164; cf. aussi pp. 123, 188, 212-213; pp. 177, 134, 203-204 et 230-232 de la traduction française.

(॰) L. c., p. 213 ; p. 232 de la traduction française. 
plement, sous une forme littéraire, le contenu de deux cours élaborés par Husserl dès $1896^{\text {(10) }}$.

Cependant, nous pouvons lire aussi, dans ce même ouvrage, des passages comme celui-ci qui fait la louange de Bernhard Bolzano: "Chez Bolzano, pourtant un contemporain de Hegel, nous ne trouvons rien de ces ambiguïtés mystiques d'une philosophie systématisée qui, au lieu de poursuivre l'idéal d'un savoir théorique et analytique du monde, s'attache surtout à celui d'une sagesse et d'une vision du monde ${ }^{111 h}$ riches en pensées profondes, et qui confond en outre malencontreusement ces deux intentions fondamentalement différentes l'une de l'autre et fait ainsi obstacle au progrès d'une philosophie scientifique " ${ }^{(12)}$. Et Husserl de mettre en garde contre "les préjugés et les façons de penser et de parler des philosophes issus des écoles idéalistes - préjugés et manières dont nous ne nous sommes pas tous encore entièrement libérés " ${ }^{(12)}$. Langage étrange, dira-t-on, de la part de celui qui est en train d'exposer ses " intentions idéalistes en logique " et de se réclamer de l'autorité de Kant.

Toutefois, en 1910-1911 encore, Husserl semble toujours juger la philosophie de l'idéalisme allemand comme Brentano le lui avait appris, c'est-à-dire en fonction d'un idéal énoncé dans le titre même de l'article qu'il publie à ce moment: Philosophie als strenge Wissenschaft ${ }^{(13)}$. Il est vrai qu'il admet, à présent, que cet idéal " se renouvelle vigoureusement et radicalement dans la critique de la raison d'un Kant et inspire toujours la philosophie de Fichte "(14). Cependant, " un changement se produit à ce propos dans la philosophie du romantisme. Il est vrai que Hegel aussi insiste sur la validité absolue de sa méthode et de sa doctrine, mais en fait, il manque à son système une critique de la raison qui seule peut rendre possible une philosophie véritablement scientifique. En conséquence, cette philosophie [hégélienne], comme la philosophie

(30) L. c., préface pour la $2^{\theta}$ édition $(1913)$, p. XII; p. XV de la traduction française.

(11) Weltweisheit und Weltanschauung.

(12) L. c., p. 266 ; p. 244 de la traduction française.

(13) Logos, I (1910-1911), pp. 289-341; traduction française avec introduction et commentaire par Quentin LAUER: E. HUSSERL, La philosophie comme science rigoureuse, 1955.

(14) Logos, I, p. 292. On trouve en marge de la traduction française la mention des pages correspondantes dans Logos. 
romantique en général, agira par la suite dans le sens, soit d'un affaiblissement, soit d'une dégénérescence des tendances vers la constitution d'une science philosophique rigoureuse " ${ }^{(14)}$. Ainsi, Hegel et la philosophie de l'idéalisme "romantique " sont rendus responsables, directement ou indirectement, de la dégénérescence de la philosophie en un empirisme sceptique d'une part, et d'autre part de l'affaiblissement de l'aspiration de la philosophie à un savoir véritablement scientifique, affaiblissement qui résulta de l'historicisme.

Du moins en ce qui concerne l'idéalisme postfichtéen, Husserl semble donc continuer de partager l'opinion de Brentano selon laquelle "les systèmes de l'idéalisme allemand " ne témoignent que d'une " décadence " de la philosophie. Il est vrai qu'en même temps et paradoxalement, il attribue indirectement à cette " dégénérescence " de la philosophie qui fut la conséquence du hégélianisme cette autre opinion de Brentano selon laquelle l'idéal d'une philosophie comme science rigoureuse était représenté par les sciences exactes de la nature.

Or, un troisième témoignage personnel de Husserl nous confirme que le changement de sa position vis-à-vis de l'idéalisme du $\mathrm{XIX}^{\mathrm{C}}$ siècle ne s'est pas accompli d'un seul coup. En effet, dans une lettre qu'il adressait, le 29 juin 1918, au philosophe néokantien Paul Natorp (15), Husserl écrit ceci: "Quoique l'influence de Kant et de l'idéalisme allemand sur moi fût restreinte dans la période de ma formation, période où j'étais comme aveugle à l'égard de la signification intime de l'un et de l'autre, mon évolution ultérieure devait m'approcher de plus en plus de cet idéalisme... Quels que fussent les chemins que je franchis, en solus ipse entièrement solitaire, à travers un terrain impraticable, chacun de ces chemins aboutit, après avoir traversé le milieu d'un Platonisme (peut-être modifié), à des sommets d'où s'ouvrirent des perspectives qui, du coup, me rendirent Kant accessible et qui, par la suite, me rendirent compréhensibles le sens profond de l'évolution de l'idéalisme allemand et la signification absolue des intentions directrices de celui-ci. En même temps, je fus certain qu'en fait, les lignes directrices immanentes à mon travail phénoménologique et rigoureusement scientifique... visaient des fins prédessinées par les intuitions ${ }^{(16)}$ de

(15) Original conservé aux Archives-Husserl à Louvain ( $R$ I Natorp).

(19) Il faut entendre ici le mot d'intuition (Intuition) dans son acception vul- 
cet idéalisme. Depuis, tout mon travail systématique se rapporte en pleine conscience à ces intuitions (18) directrices de l'idéalisme allemand, quoique cela ne soit guère manifeste dans mes écrits publiés jusqu'à présent. (A cette occasion, je me permets d'ajouter la remarque suivante: Il y a plus d'une dizaine d'années déjà que j'ai dépassé le stade d'un Platonisme statique et que j'ai fixé, comme thème principal de la phénoménologie, l'idée d'une genèse transcendantale) $)$.

La date principale que nous cherchons à déterminer, celle où Husserl prit conscience de la "parenté essentielle " entre la philosophie phénoménologique et l'idéalisme allemand inauguré par Fichte, commence à se préciser: elle devra se situer entre l'époque où fut rédigé l'article sur la Philosophie als strenge Wissenschaft (1910-1911) et l'année 1918.

D'autre part, tout en établissant un rapport étroit entre l'évolution de ses relations à la philosophie de l'idéalisme allemand et celle qu'a prise sa pensée systématique en elle-même, Husserl nous avertit que, dans ses publications antérieures à 1918, nous ne trouverons guère de traces du changement de son attitude à l'égard de cet idéalisme du $\mathrm{XIX}^{e}$ siècle. Nous ne pouvons que le confirmer.

\section{II}

Après une période de formation pendant laquelle, sous l'influence de Brentano, Husserl s'oppose fortement à la philosophie transcendantale de Kant et davantage encore à celle de l'idéalisme postkantien ${ }^{(17)}$, ses recherches l'approchent " $d$ 'un Platonisme (peut-

gaire et préphénoménologique. A d'autres occasions, Husserl parlera, plus correctement, pour désigner les vérités anticipées par les philosophes du passé, de leurs aperçus (en français dans l'original); cf., par exemple, t. VIII, p. 190. En effet, ce mot d'aperçu est apparenté à la notion d'aperception (Apperzeption) qui implique, chez Husserl, une présence médiate et préintuitive ou anticipative.

(1i) C'est sans doute à cette « période de formation » que se rapporte un passage d'une lettre adressée par Husserl à Marvin Farber et que celui-ci a reproduit, en traduction anglaise, dans son cuvre The Foundation of Phenomenology, 1943: «As a young beginner ... I was always far removed from Kantianism and German idealism. Only Natorp interested me, more for personal reasons ... (p. 17). Les relations personnelles de Husserl avec Natorp datent d'avant 1894 déjà. La date à laquelle cette lettre fut écrite n'est pas précisée par Marvin Farber. On ne peut la déterminer que d'une façon approximative; en effet, il y est fait mention de la publication imminente, dans le Philosophen-Lexikon 
être modifié) " qui s'exprimera surtout dans les Prolegomena zu einer reinen Logik, ouvrage dont l'essentiel est conçu dès 1896. Du coup, et ces Prolegomena en témoignent, un premier accès lui est ouvert à la pensée de Kant. En fait, il a commencé à étudier de

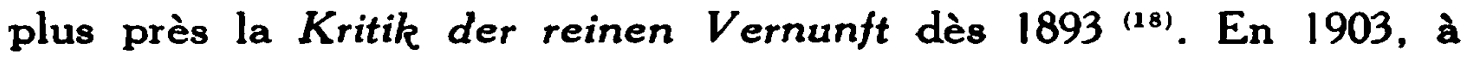
Gottingue, il consacre pour la première fois, dans un cours sur l'histoire de la philosophie, quelques leçons à la philosophie de Kant (19). Dès 1906, Husserl adoptera même une formule kantienne pour désigner "le problème général que je dois résoudre pour moimême si je veux pouvoir m appeler philosophe. Je veux parler d'une Critique de la raison " (20). La résonnance kantienne est frappante dans les Fünf Vorlesungen qui exposent, en 1907, Die Idee der Phänomenologie (21). M. Walter Biemel, l'éditeur de ce texte posthume, fait remarquer dans son introduction que, manifestement, à ce moment-là déjà, et donc bien avant l'époque de la publication des Ideen zu einer reinen Phänomenologie, "le passage de Husserl à l'idéalisme " s'est accompli ${ }^{(22)}$. On précisera, à l'appui de ce que nous lisions dans la lettre de Husserl à Natorp du 29 juin 1918. que dès 1907, l'idéalisme husserlien, platonisant, d'abord, à l'époque des Logische Untersuchungen, commence à " dépasser ce stade d'un Platonisme statique " pour se transformer en un idéalisme transcendantal fondé par une phénoménologie génétique ${ }^{(23)}$.

En effet. Husserl n'a jamais reconnu une rupture, dans son évolution philosophique, entre une période $d$ '" objectivisme" et de "réalisme" (platonisant) - celle des Logische Untersuchungen - et une période subséquente d'idéalisme transcendantal. Au contraire, pour lui, ses convictions furent idéalistes, d'une façon géné-

de W. Ziecenfuss, d'un article sur Husserl; or cet article fut rédigé par Husserl lui-même en collaboration avec Eugen Fink ou par ce dernier seul, vers 1930. (18) Cf. les notes personnelles de Husserl dans Tagebuch zum Raumbuch, journal philosophique conservé aux Archives-Husserl à Louvain (X $X$ 3).

(19) T. VII, pp. 350-356 (Beilage $X V$ ); cf. les notes de l'éditeur p. 459.

(20) E. Husserl, Persönliche Aufzeichnungen, publiées par Walter BIEMEL. dans Philosophy and Phenomenological Research, XVI (1956). P. 297 ; traduction française par Jean LAdRIERE et Walter BiEmel, dans Esprit et Vie, février 1951, p. 4. (21) T. II.

(22) T. II, p. $x$.

(2s) D'autre part, nous ferons des réserves en ce qui concerne l'hypothèse de Walter Biemel selon laquelle une étude approfondie de Kant aurait été à l'origine de cette transformation de la pensée de Husserl; l'hypothèse contraire nous semble plus proche de la vérité historique. 
rale, dès qu'il fut arrivé à la position qu'expriment ses Logische Untersuchungen, c'est-à-dire dès les années 1896-1900. Seulement, au cours des années suivantes, cet idéalisme s'est accentué dans le sens d'un idéalisme transcendantal et génétique, se rapprochant par là des conceptions de l'idéalisme moderne de Kant et de ses successeurs.

Nous en trouvons le témoignage éclatant dans l'œuvre husserlienne qui manifesta pour la première fois cette position nouvelle du philosophe qu'on vise généralement quand on parle de son " idéalisme ", les Ideen zu einer reinen Phänomenologie und phänomenologische Philosophie, dont un premier volume seulement Allgemeine Einführung in die reine Phänomenologie ${ }^{24)}$ - parut en 1913. Pour s'en rendre compte, il suffit de jeter un coup d'œil, dans le Registre élaboré par Gerda Walther, qui accompagna la $2^{\circ}$ édition de l'ouvrage $(1922)^{(25)}$, sur l'article Idéalisme: sauf quelques passages qui font allusion à l'idéalisme berkeleyien, Husserl ne parle d' idéalisme " dans cet ouvrage de 1913, qu'en visant par là, précisément, l'idéalisme " platonisant" des Logische Untersuchungen; position dont il demeure, par ailleurs, le partisan convaincu. D'autre part, il rejette explicitement, en des formules qui rappellent celles de l'article Philosophie als strenge Wissenschaft, le reproche qu on lui adressait déjà, de retomber par là dans " une 'extravagance d'idéologue', une 'régression à la scolastique' ou à ces sortes de 'constructions spéculatives $a$ prior $i$ ' par lesquelles l'idéalisme de la première moitié du XIX $\mathrm{X}^{\ominus}$ siècle, philosophie étrangère aux sciences de la nature, a tellement entravé la science authentique $)^{(26)}$.

D'autre part, Gerda Walther avait ajouté à l'article Idéalisme un autre, fort curieux, intitulé Idéalisme phénoménologique. Ce dernier était divisé en deux parties, dont l'une signalait les passages qui, dans les Ideen, favorisaient une interprétation idéaliste, l'autre

(24) T. III (dans cette édition de l'ouvrage dans le cadre des Gesammelte Werke, le texte primitif se trouve modifé par l'incorporation de notes et de corrections husserliennes qui datent d'après 1913 et même, pour la plupart, des années autour de 1922); traduction française (selon la version primitive de 1913), avec introduction et notes, par Paul RICEUR: E. HUSSERL, Idés directrices pour une phénoménologie, 1950.

(2s) Ce registre ne se trouve, sous forme d'appendice, que dans la seule édition de 1922. Il n'est repris dans aucune des éditions ultérieures des Ideen.

(28) T. III, P. 42 ; p. 64 de la traduction française. 
ceux qui semblaient s'opposer à une telle interprétation de la phénoménologie husserlienne. Dans une note, Gerda Walther expliquait que, " bien que dans les Ideen on ne parlât pas expressément d'un 'idéalisme phénoménologique', certaines affirmations husserliennes faites dans cet ouvrage auraient donné lieu à des discussions poursuivies autour de ce thème. Il nous semblait alors utile, afin de mieux orienter ces discussions, d'insérer tous les passages parlant [indirectement] pour ou contre [un idéalisme phénoménologique] dans ce registre, sous le titre qu'on trouve plus haut "(27). - Vers 1923 seulement, sous forme de notes marginales dans son exemplaire personnel (28). Husserl exprima son mécontentement du registre tel qu'il était conçu par Gerda Walther et qui reflétait pourtant, disonsle, assez exactement la situation réelle qu'on trouve dans les Ideen de 1913. Dans l'édition suivante de cet ouvrage (1928), ce registre fut alors supprimé et remplacé par un autre, moins ambigu, établi par Ludwig Landgrebe ${ }^{(29)}$; celui-ci exprimant le changement d'idées intervenu depuis dans l'esprit de Husserl.

Il est vrai que les Ideen de 1913 contiennent nombre de passages élogieux à l'égard de Kant. On y lit, par exemple: "Kant, sans doute, a perçu [le domaine d'une phénoménologie pure], Kant dont les plus grandes intuitions ${ }^{(30)}$ ne sont pleinement compréhensibles que si nous avons pris une conscience parfaitement claire des traits distinctifs du domaine phénoménologique ". Husserl y ajoute cependant: "Nous voyons alors avec évidence que Kant a posé le regard de son esprit sur cet empire quoiqu'il n'a pas pu encore en prendre possession et $y$ discerner le champ de recherche pour une science éidétique autonome et rigoureuse " (31). Et dans la même année 1913, dans l'esquisse d'une préface pour une réédition des Logische Untersuchungen, Husserl affirme toujours "que les grandes idées de la critique de la raison de Kant, redevenues accessibles grâce à l'école de Marbourg et à A. Riehl, ne constituent toutefois pas des fondements authentiques, c'est-à-dire puisés dans les sources les plus originelles et les plus pures (celles de l'intuition pure); et que, par conséquent, la philosophie transcendantale kantienne ne saurait passer, ni dans sa forme originaire,

(27) P. 23 du registre.

(28) Conservé aux Archives-Husserl à Louvain.

(20) Réimprimé dans t. III. pp. 420-456.

(sn) Cf. note 16.

(31) T. III, p. 148; pp. 203-204 de la traduction française. 
ni dans ses formes renouvelées, pour une véritable philosophie première ") ${ }^{(32)}$.

Rappelons à ce propos que la première publication de Husserl où il devait parler de façon explicite d'“ idéalisme phénoménologique ", date de 1929. Dans Formale und transzendentale Logik, en effet, on trouvera, parmi d'autres passages relatifs à cette question, un paragraphe intitulé Psychologistischer und phänomenologischer ldealismus ${ }^{(33)}$. Il est fort significatif qu'ainsi Husserl oppose toujours son idéalisme phénoménologique, non pas à un objectivisme ou réalisme au sens classique, mais à ce même psychologisme auquel il opposait, en 1900 déjà, ses "intentions idéalistes en logique ". L'idéalisme psychologique n'est qu'un "mauvais idéalisme ", parce qu'il est un "lucus a non lucendo ", c'est-à-dire un "idéalisme " sans idées et sans idéaux (34). L'idéalisme "transcendantal " husserlien, lui, est considéré par son auteur, non pas comme l'antithèse d'un "réalisme " phénoménologique qu'il aurait abandonné, mais comme un développement logique et radical des intentions idéalistes qui l'inspiraient dès l'époque des Logische Untersuchungen.

On peut mettre en doute cette continuité dans l'évolution de l'idéalisme phénoménologique de Husserl. On ne peut pas négliger les affirmations de Husserl lui-même qui la soulignent. On ne le peut, du moins, si l'on $s^{\prime}$ attache à une recherche de ce que fut la conception réellement et historiquement sous-jacente à l'essor de la pensée husserlienne, et si l'on ne se contente pas de ne chercher, en Husserl, qu'un appui d'autorité pour des convictions qu'on n'a pas le courage de défendre personnellement. Quant à nous, nous croyons qu il importe bien plus au travail philosophique lui-même de rendre intelligible la signification réelle de l'histoire des philosophies que de créer sans cesse de nouvelles "convictions " personnelles qui, en outre, ne représentent très souvent que des opinions simplement "possibles", du fait que ceux qui nous les soumettent, tout en étant leurs inventeurs, $n^{\circ}$ en paraissent pas plus

(32) E. HussERL, Entwurf einer 'Vorrede' zu den 'Logischen Untersuchungen' (1913). publié par Eugen Fink dans Tijdschrift voor Philosophie, I (1939), pp. 106133 et 319-339, pp. $110-111$.

(ss) E. HUSSERL, Formale und transzendentale Logik, 1929, \& 66, p. 151; traduction française par Suzanne BACHELARD: E. HusserL, Logique formelle et logique transcendantale, 1957.

(31) L. c., pp. 151-152. 
convaincus eux-mêmes ; ils les attribuent - ou feignent de les attribuer - à des philosophes du passé dont, en réalité, la pensée réelle ne les intéresse guère.

Revenons-en à l'évolution de l'attitude adoptée par Husserl à l'égard de la philosophie de l'idéalisme classique. Les témoignages autobiographiques dont nous cherchons à vérifier les affirmations nous disaient qu'un changement important est intervenu dans l'esprit de Husserl, à ce propos, avant même que ce changement n'eût trouvé une expression dans les écrits publiés par lui avant 1918. Devrons-nous en conclure que dans les Ideen de 1913, ce changement, déjà réel, ait été volontairement dissimulé par l'auteux ? Nous croyons, au contraire, que Husserl veut nous faire comprendre veut faire comprendre à Natorp - que ce changement s'est produit, en réalité, avant même que lui, Husserl, s'en soit consciemment rendu compte. Or, ce qui importe pour nous, c'est précisément de connaître le moment où eut lieu cette prise de conscience explicite, dans l'esprit de Husserl. Cette date, faudra-t-il donc la situer aux années de la grande guerre de 1914-1918?

Faudrait-il s'en étonner ? "Dans les années de guerre, il me fut impossible de conserver, pour la phénoménologie du logique, un intérêt passionné tel qu'il me le faut pour tout travail fructueux. Voici ce qui seul pouvait rendre supportable pour moi la guerre et la 'paix' qui la suivit: des réflexions philosophiques de l'ordre le plus général, une reprise de mes travaux consacrés à l'élaboration méthodique et concrète de l'idée d'une philosophie phénoménologique, à la conception systématique de ses lignes fondamentales, à [l'établissement de] la hiérarchie de ses objectifs de travail et à la poursuite de toutes les recherches concrètes qui semblaient indispensables en cet ordre d'idées ". C'est ce que Husserl écrit en octobre $1920^{(35)}$.

Pas plus tard qu'en 1915, Husserl parle à ses étudiants, à Goettingue, de "la philosophie idéaliste postkantienne ", dans les termes suivants: "Un jour, vous remarquerez vous-mêmes que la phénoménologie est capable de et destinée à rétablir des ponts

(ss) E. HUSSERL, Logische Untersuchungen, préface pour la $2^{\mathrm{e}}$ édition (1921) de la $2^{\circ}$ partie $\left(=6^{\circ}\right.$ Untersuchung $)$ du second volume, p. III. 
rompus, en nous permettant de nous approprier à nouveau les grandes valeurs spirituelles des philosophies idéalistes... " (38). Le document décisif, témoignant $\mathrm{du}$ changement accompli, est alors le manuscrit, conservé aux Archives-Husserl à Louvain ${ }^{(37)}$, qui contient le texte de trois conférences, faites par Husserl en 1917 et répétées en 1918, à l'intention d'étudiants mobilisés en permission, sur Fichtes Menschheitsideal, l'idéal fichtéen de l'humanité. Husserl y confirme que ce fut l'événement de la guerre qui devait donner lieu à un examen de conscience qui devait faire reconnaître à nouveau la signification profonde et la part de vérité absolue contenues dans l'idéalisme classique de la philosophie allemande du XIX siècle, celle de Fichte, de Schelling, de Hegel et même de Schleiermacher et de Schopenhauer, dont les noms sont également cités par Husserl.

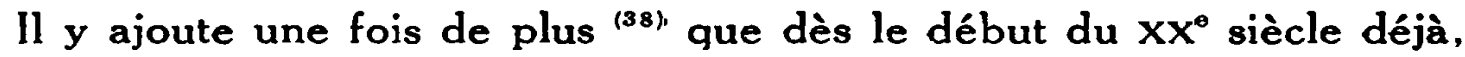
une compréhension renouvelée et une revalorisation de la philosophie de l'idéalisme allemand se préparaient et s'annonçaient ${ }^{\left({ }^{(39)} \text {. }\right.}$

Ce changement éclatant, survenu au cours des années de la guerre de 1914-1918, dans le jugement de Husserl sur la valeur de l'idéalisme allemand, n'aboutira point, cependant, à une approbation inconditionnelle et sans réserves de cette philosophie; bien au contraire. Sur les réserves importantes faites par Husserl à ce propos et dont l'analyse nous servira par la suite à saisir la différence profonde aperçue par Husserl entre l'idéalisme classique et son propre idéalisme phénoménologique, les Erinnerungen an Franz Brentano nous éclairent nettement. Brentano, dit Husserl dans le passage déjà cité par nous, "n'avait que peu d'estime pour des penseurs tels que Kant et les idéalistes allemands postkantiens, tous bien plus distingués par la valeur de leurs intuitions ${ }^{(40)}$ originelles et de leurs pressentiments anticipatifs que par celle de leur méthode logique et de leurs théories scientifiques. Qu'un penseur philosophique puisse être grand, bien que toutes ses théories, prises à la

(3s) T. VII, p. 406 (Beilage XXI).

(3r) FI 22.

(38) Cf. la lettre à Natorp, de 1918, citée plus haut.

(as) Le fait, en lui-même étrange, qu'en 1919. dans ses Erinnerungen an Franz Brentano, Husserl souligne si fortement son attachement à une philosophie dont l'influence de Brentano tendait, au contraire, à l'éloigner, s'explique alors par le rapprochement des dates: son changement d'idées à ce sujet est tout récent à ce moment-là.

(40) Cf. note 16 . 
rigueur, soient dépourvues de fondements scientifiques et que même ses concepts fondamentaux ne soient 'ni clairs ni distincts' ; que la grandeur d'un philosophe puisse résider, non dans la perfection logique de ses théories, mais dans l'originalité d'intuitions fondamentales de la plus haute importance, bien que celles-ci soient encore vagues et peu claires; résider donc dans des entéléchies encore prélogiques, puisqu'elles sont, précisément, à la recherche de leur logos, bref, dans des motivations de la pensée entièrement nouvelles et qui décideront, en dernière analyse, du sens final de tout travail philosophique, bien que, encore une fois, ces motivations soient loin encore d'être saisies et réalisées sous forme de théories rigoureuses: tout cela, Brentano l'aurait difficilement admis " (41). Voilà donc ce que seul Husserl est prêt d'admettre dorénavant. Et donc, aussi après avoir corrigé, en ce sens, son jugement sur la philosophie de l'idéalisme allemand du XIX siècle, Husserl est loin de reconnaître à cette philosophie un rang scientifique qu'il lui a toujours contesté. Sur ce dernier point, il restera donc d'accord avec Brentano.

La lettre à Natorp de 1918 dont il a été question plus haut, exprime, elle aussi, ces réserves sous une forme différente, mais non moins nette. Cette lettre fut la réponse de Husserl à l'envoi, par Natorp, d'un ouvrage de ce dernier, intitulé Deutscher Weltberuf (42). Husserl en dit beaucoup de bien et conclut ainsi : "Votre cuvre ${ }^{(43)}$ est d'une valeur inestimable... comme expression d'une vision du monde ${ }^{(44)}$ qui seule peut nous rendre possible, moi aussi j'en suis persuadé, une 'vie bienheureuse'". Manifestement, le mot-clé de ce passage est celui de "Weltanschauung ", c'est-à-dire "idéologie " ou, littéralement, "vision du monde ". En effet, déjà l'article sur la Philosophie als strenge Wissenschaft, la philosophie comme science rigoureuse, opposait celle-ci à une philosophie qui

(41) L. c., p. 159.

(62) Paul NATORP, Deutscher Weltberuf, 1918. L'exemplaire qui a appartenu à Husserl semble malheureusement perdu; il ne se trouve pas dans la bibliothèque philosophique de Husserl, conservée aux Archives-Husserl à Louvain.

(4s) Werk: ce qui peut signifier, en allemand, l'ouvrage (c'est-à-dire, dans notre cas, l'ouvrage de Natorp mentionné dans la note précédente) ou bien aussi l'ceuvre (dans ce cas, il se peut que Husserl veuille parler de l'oeuvre philosophique de Natorp dans son intégralité).

(41) Weltanschauung. 
n'exprime qu'une Weltanschauung ou idéologie (45). Nous verrons plus loin que cette parole adressée par Husserl à Natorp peut être considérée comme étant adressée, de fait, à la philosophie classique de l'idéalisme allemand tout entière, philosophie dont Natorp. pour Husserl du moins, fut le dernier grand représentant authentique. Enfin, ce n'est pas par hasard que, dans le passage cité, Husserl emploie cette expression de "vie bienheureuse ", tirée du titre d'un écrit de Fichte et sans doute entendue ici, par Husserl, au sens fichtéen ${ }^{(46)}$.

Or, ces réserves critiques constamment maintenues par Husserl à l'égard de la valeur scientifique de la philosophie de l'idéalisme allemand pourraient même sembler remettre en cause nos constatations antérieures. En effet, le jugement de Husserl sur cette philosophie ou idéologie, tel qu'il se trouve exprimé en 1915, en 1917, en 1918 et en 1919, n'est peut-être pas tellement différent de celui que l'on trouvait dans l'article Philosophie als strenge Wissenschaft, de 1910-1911. De toute façon, afin de mieux saisir la signification exacte du changement intervenu dans les rapports de Husserl à la pensée de l'idéalisme classique pendant les années de la grande guerre, il nous faudra examiner de plus près les thèses de cet article.

\section{IV}

En fait, dans Philosophie als strenge Wissenschaft, la position prise par Husserl par rapport à Hegel, d'une part, et à une philosophie de caractère purement idéologique en général, d'autre part, n'est pas sans ambiguïté.

Cette ambiguïté apparaît déjà dans cette phrase sur Hegel et

(4s) La suite du présent exposé pourra seule justifier notre traduction, du reste fort commode, du mot allemand Weltanschauung (vision du monde, conception du monde), chez Husserl, par idéologie. Nous sommes conscient du fait que nous négligeons les rapports de Husserl à Dilthey qui s'expriment dans l'article Philosophie als strenge Wissenschaft: en effet, le mot Weltanschauung fut introduit dans la terminologie philosophique par Dilthey.

Pour ce qui concerne lesdits rapports, cf. Dilthey-Husserl. En torno a la Filosofia como Ciencia estricta y al alcance del historicismo. Correspondencia entre Dilthey y Husserl, publiée, en allemand et en espagnol, avec introduction et notes, par Walter BIEMEL, dans Revista de Filosofia de la Universidad de Costa Rica, I (1957). pp. 101-124.

(46) J. G. Fichte. Anweisung zum seligen Leben, 1806. 
l'idéalisme "romantique " que nous avons déjà citée: "ll est vrai que Hegel aussi insiste sur la validité absolue de sa méthode et de sa doctrine, mais en fait, il manque à son système une critique de la raison qui seule peut rendre possible une philosophie véritablement scientifique " ${ }^{(47)}$. La philosophie hégélienne demeure donc une simple idéologie bien qu' elle aspire à une validité absolue de sa méthode et de sa doctrine; et elle insiste sur la validité d'un prétendu savoir absolu bien qu'elle ne puisse être elle-même qu'une idéologie, et non une philosophie véritablement scientifique ${ }^{(48)}$. Elle sera donc l'idéologie d'une philosophie comme science rigoureuse.

Or, une ambiguïté absolument semblable se manifeste, d'autre part, dans la position que Husserl adopte sur le problème de l'idéologie philosophique, dans Philosophie als strenge $W$ issenschaft.

L'article tout entier exprime solennellement le parti pris par son auteur en faveur de l'idée d'une philosophie comme science rigoureuse, et contre celle, ambitieuse et résignée à la fois, d'une philosophie de caractère idéologique ; à cette dernière idée, Husserl concède un rôle important et une valeur réelle, tout en réservant à la seule philosophie comme science rigoureuse une "valeur absolue ". Or, en fait, cette prise de position elle-même ne peut relever que d'une motivation d'ordre idéologique: "il s'agit là encore, admettra Husserl, d'une question pratique " (48) - et non point d'un problème d'emblée susceptible d'une solution théorique et scientifique. En effet, la question touchant les valeurs respectives qu'on doit attribuer à l'idéal d'une philosophie comme science théorique rigoureuse, d'une part, et à une philosophie comme idéologie inspirée par les besoins pratiques de l'humanité, d'autre part, doit demeurer elle-même une question d'ordre purement idéologique tant qu'une philosophie comme science rigoureuse n'existe même pas encore. Et de fait, c'est cela que Husserl souligne expressément dès le

(4) Logos, I, p. 292.

(4s) Husserl doit $s^{\prime}$ être rendu compte de l'ambiguïté de cette situation lorsqu'il notait, au moment où il travaillait à l'article Philosophie als strenge Wissenschaft, les définitions de la philosophie comme "science absolue " et a science de l'absolu 2 proposées par Schelling et Hegel (cf. t. VII, p. 310). Plus tard, vers 1924, Husserl admettra même que, dans la Phänomenologie des Geistes, a Hegel tente de mettre en lumière comment l'esprit humain est poussé, à partir d'une conception naive du monde et de la vie (naive Welt- und Lebensauffassung), et par les contradictions immanentes à celle-ci, au point de vue de la philosophie (t. VII, p. 312, note). (49) Logos, I, p. 334. 
début de l'article: "Je ne dis pas que la philosophie soit une science imparfaite, je dis tout simplement qu'elle $n^{\prime}$ est point encore une science, qu'elle n'a pas encore fait son début comme science, et en cela je prends pour critère n'importe quel fragment, si petit soit-il, d'un contenu doctrinal objectivement établi " ${ }^{(50)}$.

Or, cette situation, ne justifierait-elle point de répéter, au sujet de Husserl lui-même, ce que celui-ci disait au sujet de Hegel ? $\mathrm{Ne}$ pourrions-nous retourner ainsi cette affirmation husserlienne qui visait Hegel: "Il est vrai que Husserl aussi insiste sur la validité absolue que doivent acquérir la méthode et la doctrine d'une philosophie (comme science rigoureuse), mais en fait, il lui manque toujours cette critique de la raison qui seule peut rendre possible une philosophie véritablement scientifique " (51) ? Qu'est-ce donc que la conception husserlienne exposée dans l'article que nous analysons sinon une nouvelle version de l'idéologie d'une philosophie comme science rigoureuse ?

Cette situation, apparemment paradoxale et néanmoins fort simple en réalité, établit entre l'idéologie philosophique et l'idée d'une philosophie scientifique un rapport qui remettra en question une thèse husserlienne qui occupe pourtant une place centrale dans cet article: à savoir que, "pour la conscience moderne, les idées de culture ou d'idéologie et de science - entendues comme idées pratiques - se sont radicalement séparées, et dorénavant elles resteront séparées pour toute l'éternité " ${ }^{(52)}$. Il est vrai qu'une page plus loin déjà. Husserl lui-même reprendra cette thèse sous une forme légèrement, mais sensiblement modifiée: "Ainsi se sont radicalement distinguées: la philosophie comme idéologie et la philosophie scientifique, comme deux idées qu'il ne faut pas mêler, bien que certaines relations existent entre elles" (5s). Et même: "On peut dire également que les réalisations de ces idées (une possibilité de réalisation supposée pour chacune d'elles) s'approcheraient l'une

(80) L. c., p. 290.

(51) Les textes de Philosophie als strenge Wissenschaft nous font croire que la situation de Husserl n’a pas changé depuis 1906, c'est-à-dire depuis le moment où il avouait ne pouvoir s'appeler philosophe, étant donné qu'il n'avait pas encore résolu le problème général d'une critique de la raison (voir plus haut et note 20). En réalité, nous aurons à corriger quelque peu cette impression que donne Philosophie als strenge Wissenschaft (voir plus loin et note 59).

(82) Logos, I, p. 332.

(8s) L. c., p. 333; nous soulignons. 
de l'autre, asymptotiquement, et se correspondraient, pour autant que nous voudrions nous représenter fictivement l'illimité de la science comme un 'point infiniment éloigné " ${ }^{154)}$. En réalité, cependant, il faudra admettre l'existence d'un rapport plus concret et plus substantiel encore entre l'idéologie et la philosophie scientifique :

1) La philosophie scientifique elle-même ne peut prendre son essor que grâce à l'existence préalable d'une idéologie déterminée, de celle précisément qui prend parti pour l'idée d'une philosophie comme science rigoureuse et qui aura pour tendance, par conséquent, de dépasser son propre statut de simple idéologie. Ce qui, dans Philosophie als strenge Wissenschaft, est mis en valeur par Husserl, c'est en fait une telle idéologie préliminaire à une philosophie scientifique.

2) Seule la réalisation effective d'une philosophie comme science rigoureuse peut légitimer les idées d'une idéologie qui a voulu la promouvoir. Autrement dit, la philosophie réalisée sous forme de science rigoureuse, et elle seule, pourra et devra fournir un critère valable qui, pour toute époque du passé, du présent et même d'un avenir où la réalisation de cette philosophie scientifique, bien qu'éventuellement déjà mise en œuvre, ne sera pas encore achevée, servira à distinguer idéologies authentiques (c'est-à-dire anticipativement " vraies") et inauthentiques (" fausses", " erronées ").

Concrètement, ces rapports essentiels et nécessaires qui existent entre l'idéologie et la philosophie scientifique seront donc des rapports historiques et dialectiques ${ }^{\text {(5) }}$.

Historiquement, à un moment où la philosophie " n'a même pas encore fait son début comme science ", la tendance vers une philosophie scientifique ne peut prendre racine que dans une idéologie ; d'autre part, les progrès réels que fera une philosophie rigoureusement scientifique rendront légitime une idéologie déterminée qui précédait cette philosophie: une idéologie que la science fera comprendre comme l'anticipation prélogique ("intuitive") d'une philosophie authentique. Et Husserl le dit: "On voit clairement comment la décision en question [celle, à savoir, de s'en tenir à la sagesse pratique d'une idéologie ou au contraire à une philo-

(sa) Ib.

(55) Pour ce qui concerne le caractère proprement dialectique de ces rapports, voir plus loin. 
sophie comme science rigoureuse] se poserait ..., si les commencements indubitables d'une doctrine philosophique étaient déjà présents. Jetons un coup $d$ 'œil sur $d$ 'autres sciences. Dans le domaine des mathématiques et des sciences de la nature, toute 'sagesse' et toute doctrine de la sagesse naturelle ont perdu leur droit dans la mesure où la doctrine théorique correspondante est fondée d'une manière objectivement valable. La science a parlé ; dorénavant, il revient à la sagesse d'apprendre. L'aspiration, dans le cadre des sciences de la nature. à une sagesse n'était pas, avant l'existence d'une science rigoureuse, injustifiée; elle n'est pas, après coup, discréditée pour son époque " ${ }^{(58)}$. Cependant: "La situation n'est pas exactement la même en ce qui concerne la philosophie scientifique parce que, justement, on $n^{\prime}$ a même pas encore établi, ici, les bases d'une doctrine scientifiquement rigoureuse " (57). Ce qui revient à admettre que la décision en faveur du travail pour une philosophie comme science rigoureuse ne peut se fonder, provisoirement, que sur des considérations qui sont elles-mêmes encore d'un ordre purement idéologique.

Et pourtant, ces réflexions husserliennes touchant les rapports entre idéologie et philosophie scientifique jettent elles-mêmes les bases théoriques pour une réconciliation, imminente de fait, du philosophe avec celle des idéologies du passé qu'il devra reconnâtre comme un présupposé historique indispensable pour l'avenir d'une philosophie comme science rigoureuse; idéologie qui se trouvera légitimée, par l'instauration de cette philosophie comme science rigoureuse, dans " la valeur de ses intuitions originelles et de ses pressentiments anticipatifs", dans sa "grandeur", dans "l'originalité d'intuitions fondamentales de la plus haute importance ", dans " ses entéléchies encore prélogiques, parce qu'elles sont précisément à la recherche de leur. logos, bref, dans des motivations de la pensée entièrement nouvelles et qui auront décidé, en dernière analyse, du sens final du travail philosophique " - toutes expressions husserliennes tirées des Erinnerungen an Brentano de 1919.

Ce n'en est qu'une des conséquences de la nature dialectique des rapports historiques et concrets entre l'idéalisme idéologique et l'idée d'une philosophie scientifique que, aussi longtemps que cette philosophie "n'a pas encore fait ses débuts comme science".

(so) Logos, I, p. 334.

(nz) L. c., p. 335. 
c'est-à-dire dans la situation historique décrite dans Philosophie als strenge Wissenschaft, une prise de position en faveur de l'idée d'une philosophie scientifique doit se diriger nécessairement contre toute idéologie en général qui, en tant que telle, est contraire à l'idéal scientifique. Paradoxalement, cette prise de position doit donc se diriger aussi contre une idéologie qui, elle, est "l'idéologie d'une philosophie scientifique ". Pour combler le paradoxe, cette dernière idéologie elle-même est seule à constituer le fondement de cette prise de position - une philosophie scientifique n'ayant même pas encore fait ses débuts. La philosophie scientifique a ses origines anticipatives dans une idéologie déterminée qui consiste à rejeter toute idéologie en général et en tant que telle et qui implique donc le refus d'elle-même. Cette contradiction est immanente à l'idéologie de l'idéalisme classique si le rôle de celui-ci est tel que Husserl semble le concevoir dès qu'il écrit les lignes sur Hegel dont nous avons relevé l'ambiguité. Le contenu, les doctrines idéologiques de cette philosophie, sa " sagesse ", exigent une suppression progressive de ses propres fondements en tant qu'ils ne sont eux-mêmes que des fondements idéologiques. Cette idéologie demande une réalisation qui ne pourra consister que dans une suppression et un dépassement d'elle-même en tant qu idéologie ${ }^{(58)}$.

Telles sont, sans doute, les origines des rapports, toujours ambigus, de la philosophie phénoménologique de Husserl à l'idéalisme allemand classique.

\section{V}

Il est vrai que, une fois jetées les bases d'une philosophie

(58) On remarquera que, pour caractériser la situation qui est à la base des rapports de Husserl à la philosophie de l'idéalisme classique, nous avons employé des expressions empruntées à l'article Zur Kritik der Hegelschen Rechtsphilosophie du jeune Marx; selon ce dernier, en effet, on ne peut réaliser la philosophie (de l'idéalisme classique) sans la supprimer et la dépasser, comme on ne peut non plus la supprimer ou la dépasser sans la réaliser (cf. Karl MARX - Friedrich ENGELs, Werke, nouvelle édition de Berlin, t. I, 1957, p. 384). Les différences évidentes qui existent entre les positions adoptées, vis-à-vis de l'idéalisme allemand classique, par Marx, d'une part, et par Husserl, d'autre part, n'excluent pas une analogie réelle de leurs situations respectives. Une étude comparée des deux critiques, marxiste et husserlienne, de cette a idéologie» promettrait des résultats intéressants. Nous n'apporterons ici que quelques éléments, sous forme de notes, à une telle étude. 
rigoureusement scientifique, les rapports de cette philosophie aux idéologies du passé pourront être établis, eux aussi, sur un fondement scientifique. Dès ce moment, le refus "sommaire ", lui-même de nature idéologique, de toute idéologie en général et en tant que telle, pourra faire place à une réflexion qui, partant des réalisations d'une philosophie scientifique en marche, distinguera entre idéologie et idéologie; en particulier cette réflexion rendra justice à celle des idéologies du passé dont les "entéléchies encore prélogiques,... les motivations entièrement nouvelles de la pensée auront décidé, en dernière analyse, du sens final du travail philosophique", maintenant en train de se réaliser.

Or, deux ans après l'article Philosophie als strenge Wissenschaft (publié en 1910-1911), Husserl fera paraître en 1913 la première partie d'une ceuvre par laquelle, il en était convaincu, une philosophie comme science rigoureuse faisait réellement ses débuts. Dans l'introduction à cet ouvrage, intitulé Ideen zu einer reinen Phänomenologie und phänomenologischen Philosophie, Husserl écrit : "Un troisième et dernier livre [de cet ouvrage] est consacré à l'idée de la philosophie. On y fera comprendre qu'une philosophie authentique. dont l'idée est de réaliser l'idée d'une connaissance absolue, prend racine dans une phénoménologie pure; et cela en un sens aussi rigoureux que l'instauration et l'élaboration de cette première de toutes les philosophies constituera la condition préalable et nécessaire pour toute métaphysique et toute autre philosophie - 'qui pourra se présenter comme science' $"{ }^{(59)}$.

(s9) T. III, p. 8; pp. 8-9 de la traduction française. Le dernier bout de phrase. mis entre guillemets par Husserl, reprend, on l'aura remarqué, une formule de Kant qui a écrit des Prolegomena zu einer jeden künftigen Metaphysik, die als Wissenschaft wird auftreten können, 1783.

En ce qui concerne la question de savoir à quel moment Husserl crut "avoir trouvé une méthode universelle pour édifier une philosophie comme science rigoureuse „, Edith Stein a affirmé qu'il $s^{\prime}$ en est convaincu a au cours même de son travail aux Logische Untersuchungen ». "En exposer la portée universelle et le fondement ultime, devait être l'objet des Ideen ... (cf. La Phénoménologie. Journées d'études de la Société thomiste, Juvisy, 12 septembre 1932, pp. 102 et 44).

Husserl lui-même dira (après avoir fait allusion au fait que la rédaction finale des Logische Untersuchungen fut achevée en 1899): "Bien plus tard seulement une réflexion $\mathrm{m}^{\prime a}$ apporté l'évidence ... que seule une science qui serait fondée. immédiatement, sur une 'phénoménologie transcendantale' et qui puiserait des sources originelles de celle-ci pourra répondre à l'idée plénière d'une connaissance rigoureusement légitimée» (cf. Entwurf zu einer 'Vorrede' etc., voir note 32, 
Pourtant c'est en vain que, plus haut déjà, nous avons cherché dans cette oeuvre la trace $d$ 'un changement qui serait intervenu dans les jugements de Husserl au sujet de la philosophie de l'idéalisme allemand classique. D'une manière plus générale, Husserl y adopte une attitude d'" époché philosophique " qui, "formulée en termes explicites, doit consister en ceci que nous suspendions notre jugement à l'égard de l'enseignement de toute philosophie préalable et que nous poursuivions nos analyses dans les limites imposées par cette suspension de jugement " ${ }^{(60)}$.

En fait, cela correspond très exactement à cette conclusion de Philosophie als strenge $W$ issenschaft selon laquelle " le radicalisme qui appartient à une véritable science philosophique " exige "que nous n'acceptions aucune donnée préalable, que nous ne laissions rien de traditionnel valoir comme point de départ et que nous ne nous laissions aveugler par aucun nom, quelque grand qu'il soit, mais qu'au contraire nous cherchions les commencements en nous soumettant volontairement aux problèmes mêmes et aux exigences qui naissent de ces problèmes... Il ne faut pas que l'impulsion de la recherche surgisse des philosophies mais des choses et des problèmes " ${ }^{(61)}$.

Il faudra l'admettre: Non seulement la philosophie, avant d'avoir fait son début comme science, doit s'opposer radicalement et indifféremment à toute simple idéologie, mais encore en établissant ses commencements véritablement scientifiques, elle doit s imposer cette "époché philosophique " qui lui interdira même de reconnaître, c'est-à-dire d'admettre et de respecter les valeurs authentiques et les vérités contenues dans une idéologie qui, en l'anticipant, l'aura, de quelque manière, rendue possible ${ }^{(62)}$.

p. 132). On pourra aussi comparer la préface de l'article Kant und die Idee der Transzendentalphilosophie (voir note 2).

Quoi qu'il en soit, étant donné la proximité des dates de la publication des Ideen et de celle de Philosophie als strenge Wissenschaft, le langage de Husserl, dans ce dernier écrit, semble quelque peu inspiré par une tendance à dramatiser la situation à la veille de la publication de l'autre.

(60) T. III. pp. 40-41; pp. 61-62 de la traduction française.

(c1) Logos, I, p. 340.

(62) Vers 1923 encore, Husserl soulignera que, “bien entendu, ... l'idéalisme transcendantal phénoménologique est loin de constituer le contenu intégral de !a phénoménologie, comme il n'en désigne pas non plus le thème. Il en est le résultat ...» (cf. t. VIII, p. 482, dans Beilage $X X X$ ). Que l'idéalisme transcendantal, résultat auquel aboutit la phénoménologie pure, ne puisse en constituer 
D'autre part, il est évident que cette attitude d'" époché philosophique ", c'est-à-dire l'attitude d'époché prise à l'égard des philosophies ou des idéologies du passé et de toute "idée de la philosophie " en général, attitude dont Husserl ne s'éloigne guère au cours des analyses qui remplissent les trois livres des Ideen que nous possédons aujourd'hui, aurait dû subir une modification considérable dans ce " troisième et dernier livre " qui se trouve annoncé dans le passage de l'introduction à l'ouvrage que nous venons de citer. En fait, cependant, ce "troisième et dernier livre, consacré à l'idée de la philosophie ", ne sera jamais écrit par Husserl (63). Ce n'est qu'après la fin de la guerre, au cours des années de 1922 à 1924, que Husserl devait s'attaquer, d'une manière systématique, aux problèmes qui devaient en faire l'objet ${ }^{(84)}$. Est-ce un pur hasard ?

Nous ne le croyons point. Le fait que par les Ideen, une philosophie rigoureusement scientifique fit réellement son début, remplit, certes, la condition première et indispensable pour une considération nouvelle, par le philosophe, des rapports de cette science neuve aux idéologies philosophiques du passé. Nous l'avons montré. Cependant, étant donné le style nécessaire pour un commencement de cette philosophie comme science rigoureuse, des motivations d'un ordre autre et nouveau devaient intervenir pour donner lieu à des

le thème, voire le but explicite, cela tient à une problématique de méthode propre à une philosophie comme science rigoureuse, c'est-à-dire à la méthode phénoménologique en tant que celle-ci est opposée aux procédés constructifs de l'idéalisme classique. Nous parlerons de ces problèmes de méthode à la fin de la présente étude.

(83) Husserl lui-même n’a publié en 1913 que le livre premier des Ideen. Les livres deuxième et troisième qui ont paru à titre posthume (t. IV et $V$ ) en 1952. ne constituaient, dans le plan primitif de l'œuvre, que deux parties à l'intérieur du deuxième livre. En effet, vers 1923, Husserl lui-même changea ce plan primitif qu'il avait dessiné dans l'introduction à la publication de 1913. Pour les détails de l'histoire littéraire des Ideen, on pourra lire l'excellente introduction. écrite par Marly Biemel, aux deuxième et troisième livres, édités par elle; voir $t$. IV, surtout pp. XIV sqq.

(04) Cf. le cours, fait par Husserl en 1923.1924, sur l'Erste Philosophie, publié dans les $t$. VII et VIII, ainsi que l'introduction, par l'auteur de la présente étude. a la première partie du cours; voir t. VII, surtout $p p$. XX sqq. Ce cours peut être lu a la place du troisième livre primitivement projeté pour les Ideen. On pourra se reporter aussi aux Cartesianische Meditationen (t. I), de 1929, dont il existe une traduction française, par E. Levinas et G. Pfeiffer: E. Husserl, Méditations cartésiennes, 1931. 
réflexions qui feront "remarquer " à Husserl, comme il le dira alors à ses auditeurs, "que la phénoménologie est capable de et est destinée à rétablir des ponts rompus en nous permettant de nous approprier à nouveau les grandes valeurs spirituelles des philosophies idéalistes" du XIX ${ }^{e}$ siècle allemand ${ }^{(65)}$.

\section{VI}

De quel ordre devaient être ces motivations nouvelles? $D^{\prime}$ un ordre, précisément, où peuvent apparaître la valeur particulière, la mission historique et le droit relatif des idéologies. Quelle est cette dimension? Husserl l'a dit dans Philosophie als strenge Wissenschaft; c'est le domaine de la vie et de ses besoins pratiques et sans répit, c'est le règne de l'urgence du temps: "C'est ... le besoin le plus radical de la vie que nous éprouvons, un besoin qui ne s'arrête à aucun point de notre vie. Tout vivre [en effet] est un prendre position, tout prendre position tombe sous un devoir, sous une juridiction au sujet de la validité et de la non-validité, selon des prétendues normes de valeur absolue " ${ }^{(66)}$. C'est cette nécessité absolument impérieuse de prendre position qui est à l'origine de toute idéologie. C'est elle qui en justifie, par l'urgence du besoin pratique, les enseignements qui ne sont pourtant pas fondés au point de vue d'une science rigoureuse. "ll est certain que nous ne pouvons pas attendre. Il faut que nous prenions position, que nous nous efforcions, par le moyen d'une 'idéologie' (67) rationnelle, bien que celle-ci demeure dépourvue d'un fondement scientifique, que nous nous efforcions de rendre harmonieuse notre prise de position envers la réalité - cette réalité de la vie dont la signification nous importe et dans laquelle notre être doit avoir son importance et sa signification " ${ }^{(88)}$.

Or, à l'époque où Husserl écrivait ces lignes, il concluait pourtant en disant: "Dans l'intérêt du temps, nous ne pouvons pas sacrifier l'éternité [à laquelle est destinée une philosophie rigoureusement scientifique]; pour adoucir notre besoin, nous ne pou-

(65) Voir plus haut et note 36.

(00) Logos, 1, p. 336.

(0i) Welt- und Lebensanschauung: littéralement donc a conception du monde et de la vie ».

(88) Logos, I. p. 336. 
vons pas transmettre aux générations futures le besoin et les besoins du présent qui finiront par devenir inextirpables. Le besoin ici relève de la science ") "69).

Et pourtant, quelques années plus tard seulement, et à un moment où, avec les Ideen, une philosophie comme science rigoureuse a déjà fait son début, ces besoins pratiques du temps et de la vie devaient atteindre à un degré tel qu'ils auront le pas, dans l'esprit de Husserl, sur ceux de la science philosophique elle-même qui travaille. elle, pour l'éternité: c'est la guerre. Et Husserl l'avouera, en 1917, en s'adressant à ses auditeurs, étudiants mobilisés en permission, en des paroles que voici : "Ah cette guerre !... Aujourd'hui, nos éducatrices sont la nécessité et la mort. Depuis des années, la mort n'est plus un événement exceptionnel; on ne peut plus la dissimuler sous des couronnes entassées et par des pompes solennelles et purement conventionnelles, et en travestir la majesté sévère. La mort a reconquis ses droits sacrés et originels. De nouveau, c'est elle qui, au sein du temps, nous fait penser à l'éternité ${ }^{(70)}$. Et $c^{\prime}$ 'est ainsi qu'un accès nous est rendu à l'idéalisme allemand " ${ }^{(71)}$. Ce fut la guerre, son apogée de la mort surtout, de la mort, de ce rappel suprême de l'urgence du temps, qui fit renaître, pour Husserl, un besoin auquel la science philosophique, encore à ses débuts abstraits, ne pouvait répondre. A ce moment-là, Husserl se tourna vers l'idéologie de l'idéalisme allemand. En effet, la nouvelle philosophie rigoureusement scientifique, loin d'être achevée, laissait sans réponse les questions urgentes d'un

(80) $\mathrm{lb}$., p. 337. - On se convaincra, d'autre part, que ce besoin pratique d'une idéologie ou vision du monde dont parle Husserl était sérieusement éprouvé par lui-même. En effet, en 1906, il écrivait avec chagrin: « Ma personnalité ne peut malheureusement plus atteindre à une forme parfaite. Elle ne peut plus se donner l'unité d'une vision du monde (Weltanschauung), ni celle d'une culture librement épanouie, harmonieusement et organiquement constituée „ (cf. Persönliche Aufzeichnungen, $I$. c., p. 296; p. 3 de la traduction française. $l$. c.; voir note 20).

(:0) L'éternité qui nous est rappelée par la mort est, manifestement, une autre éternité que celle pour laquelle travaille une philosophie comme science rigoureuse. L'expérience de la mort, tout en rappelant l'éternité, accentue l'urgence des besoins temporels. Le travail pour une philosophie comme science rigoureuse, au contraire, vise comme un avenir éternel et terrestre du genre humain.

(ii) Extrait du manuscrit $F I$ 22, conservé aux Archives-Husserl L Louvain; voir plus haut et note 37 . 
ordre pratique et éthique que posait à la réflexion l'événement de la première guerre mondiale.

Toutefois, cette philosophie comme science rigoureuse, parvenue à jeter ses bases premières, avait changé d'une manière essentielle les données du problème de l'idéologie. L'article de 19101911 ne fournissait qu'une légitimation relative de l'idéologie, tout en s'opposant, d'autre part, à toute idéologie en tant que telle et en général. Et l'analyse en question s'était terminée comme suit: "Les idéologies peuvent entrer en lutte; seule la science peut décider ; et sa décision porte le sceau de l'éternité " ${ }^{1721}$. Or la réalisation, fragmentaire mais fondamentale, d'une philosophie scientifique, dans les Ideen, permit à Husserl une décision en faveur d'une idéologie déterminée.

Par ailleurs, la situation paradoxale où Husserl se trouvait, au lendemain de l'achèvement du premier volume des Ideen et au début de la guerre de 1914, ressort, et plus clairement d'une manière indirecte que de façon explicite, du texte suivant, déjà cité par nous, si nous le réexaminons maintenant à la lumière des résultats acquis: "Dans les années de guerre ... voici ce qui seul pouvait rendre supportable pour moi la guerre ...: des réflexions philosophiques de l'ordre le plus général, une reprise de mes travaux consacrés à l'élaboration méthodique et concrète de l'idée d'une philosophie phénoménologique, à la conception systématique de ses lignes fondamentales, à [l'établissement de] la hiérarchie de ses objectifs de travail et à la poursuite de toutes les recherches concrètes qui semblaient indispensables en cet ordre d'idées " ${ }^{(73)}$. Husserl y dit qu'il lui fut impossible " de conserver pour la phénoménologie du logique un intérêt passionné tel " qu'il lui eût été nécessaire pour refondre intégralement ses Logische Untersuchungen ${ }^{(74)}$. La guerre l'aurait obligé à retourner son esprit vers " des

(i2) Logos, I, p. 337.

(73) Voir note 35.

(74) En effet, après avoir rédigé une première version des textes publiés en 1952 seulement comme deuxième et troisième livres des Ideen, en 1912. Husserl travailla d'abord de nouveau aux Logische Untersuchungen pour en préparer une

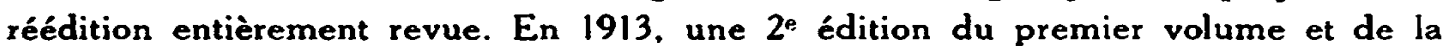
première partie (=Untersuchungen 1 à 5 ) pouvait paraître. Le début de !a guerre de 1914 trouva Husserl travaillant à une refonte intégrale de la $6^{\mathrm{e}}$ Untersuchung. Vers le début de l'année 1915, cependant, il interrompit ce travail, bien que, pour une première partie de son manuscrit, les épreuves fussent déjà composées par 
réflexions philosophiques de l'ordre le plus général ", à reprendre " ses travaux consacrés à l'élaboration méthodique et concrète de l'idée d'une philosophie phénoménologique ". En le disant, Husserl fait un aveu surprenant. En effet, ne venait-il point de publier, en 1913, le premier volume d'un ouvrage qui devait exposer les "idées directrices", non seulement "pour une phénoménologie pure", mais encore, précisément, "pour une philosophie phénoménologique " ? N'avait-il point annoncé, en publiant le premier livre de cet ouvrage, un deuxième et surtout " un troisième et dernier livre, consacré à l'idée de la philosophie " ? De ce fait, Husserl ne se sentait donc point poussé, étonnamment, à consacrer son travail " à l'élaboration méthodique et concrète de l'idée d'une philosophie phénoménologique " ${ }^{(75)}$. Pour l'y obliger, il ne fallut rien de moins que l'événement de la guerre.

Et en 1920 encore, au moment où il écrit les lignes que nous venons d'analyser, Husserl ne semble point conscient de ce qu'il y a de frappant dans la manière dont il explique les dispositions et les "obligations " qu'il ressentait durant les années de guerre. Au fait, ses explications confirment, d'une façon indirecte mais évidente. ce que nous avons voulu montrer: à savoir qu'outre le fait d'une philosophie comme science rigoureuse ayant fait son début, une motivation ultérieure était nécessaire, pour Husserl, pour l'inciter à une réflexion sur la signification historique de l'idéologie de l'idéalisme classique pour une philosophie phénoménologique comme science rigoureuse; quant aux résultats de cette réflexion, nous les connaissons déjà.

\section{VII}

A travers cette évolution mouvementée des rapports de Husserl à la philosophie classique de l'idéalisme allemand, une conviction fondamentale du fondateur de la phénoménologie ne subit aucun changement: à savoir que, avant qu'une philosophie phénoménologique au sens husserlien n'ait fait son début, cet idéalisme n’a jamais existé que sous forme d'idéologie, et non pas sous forme d'une philosophie scientifique. Il nous reste donc à examiner cette

l'imprimerie. La réédition de la $6^{\circ}$ Untersuchung qui ne parut alors qu'en 1921 ne

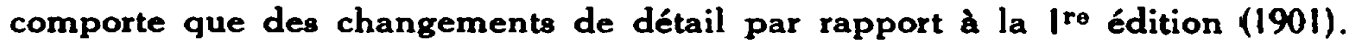

(75) Voir notes 35 et 73. 
thèse constamment maintenue par Husserl. Toutefois, nous limiterons notre analyse à la question de savoir dans quels moments distinctifs et décisifs Husserl croit découvrir le caractère d'idéologie - et donc non-scientifique - de l'idéalisme classique ${ }^{(76)}$.

Quant à savoir, d'autre part, en quoi a consisté, selon Husserl, l'apport positif de cette philosophie de Kant, de Fichte, de Schelling, de Hegel et de leurs successeurs, nous avons pu constater que, peu à peu, Husserl a pris conscience du fait que, d'une manière générale, elle constituait une anticipation, sous forme d'idéologie, d'idées fondamentales qui trouveront leur confirmation, sous une forme rigoureusement scientifigue, par cette philosophie phénoménologique qu'il a mis sur pied lui-même. Nous n'aurions rien à ajouter à cette constatation générale, si toutefois nous n'étions obligé de contredire une représentation simpliste et erronée, mais fort répandue, de ce qu'il y aurait de "commun " entre l'idéalisme allemand du $X X^{e}$ siècle et celui du XX' $X^{\circ}$, c'est-à-dire celui de Husserl. Cette opinion, elle s'exprime couramment comme suit: "Husserl est passé à l'idéalisme en mettant en avant l'idée d'une réduction phénoménologique "; et elle a pour fondement une interprétation de cette réduction qui attribue à celle-ci la signification d'une thèse qui affirmerait que "le monde réel se réduit à une subjectivité transcendantale, à une conscience absolue ". Selon cette interprétation, enfin, la phénoménologie husserlienne se serait assigné pour but, dès l'adoption de son idée de la réduction, de confirmer et de prouver la thèse en question.

Cette interprétation courante consiste donc à substituer à l'idée husserlienne d'une réduction phénoménologique ou transcendantale ce qu'on peut considérer - et qu'on considère en général - comme

(10) Nous devons renoncer, dans le cadre de la présente étude, à examiner le bien-fondé des critiques adressées par Husserl aux philosophies de lidéalisme allemand du XXe siècle. On soulèvera, d'autre part, la question de savoir si Husserl connaissait suffisamment les textes des ouvres de Kant, de Fichte, de Schelling et de Hegel pour fonder sur eux ses critiques. Avouons immédiatement, sans entrer dans le détail, qu'il ne semble avoir réellement étudié que les œuvres principales de Kant et les écrits « populaires» de Fichte. De Hegel, il n'a peut-être lu qu'une cinquantaine de pages. Ces faits ne pourront cependant dispenser d'une analyse des critiques husserliennes en elles-mêmes et d'un examen de leur portée réelle. Il faudrait aussi comparer les observations husserliennes avec celles des critiques principaux de l'idéalisme allemand du XIX siècle: Marx (voir notes 58 , 97. 98 et 108), Kierkegaard et Nietzsche. 
la thèse fondamentale de tout idéalisme ${ }^{(77)}$. Pour y répondre, suffit-il de répéter avec Husserl que le but de la recherche phénoménologique ne peut être de fournir la preuve d'une thèse idéaliste, parce que cette thèse n'en constitue même pas le thème ${ }^{(78)}$; que le monde réel ou transcendant ne se réduit point, de par lui-même, à une subjectivité transcendantale ou à une conscience absolue, parce que. au contraire, cette réduction est un travail méthodique imposé au phénoménologue; que la théorie de la réduction ne présuppose point une thèse idéaliste, parce qu'elle a pour objet, non pas une thèse à établir, mais une méthode de recherche à effectuer ?

En répétant simplement tout cela, nous ne dissipons pas les confusions. Il ne peut certainement pas être question de remettre en doute le fait que Husserl est " passé à l'idéalisme " moyennant des procédés relevant $d$ 'une méthode de réduction phénoménologique ; il ne s'agit que de préciser la signification de ce fait ou de cette affirmation. D'autre part, cette " simple précision " fera apparaître les rapports existant entre la phénoménologie husserlienne et une conception idéaliste du monde sous un jour bien différent et, peut-être, entièrement nouveau. En effet, nous aurons à montrer que, dans l'esprit de Husserl, l'idée d'une réduction transcendantale, loin de relever de ce qu'il y a de "commun" dans tout idéalisme, désigne une méthode proprement phénoménologique; et que l'absence de la conception d'une telle méthode constitue le defaut spécifique qui rendait impossible à l'idéalisme classique du $\mathrm{XIX}^{\circ}$ siècle, de se défaire jamais de son caractère de pure idéologie.

Pour l'essentiel, l'évolution des rapports de Husserl à la philosophie de l'idéalisme classique est terminée, nous l'avons dit, vers 1923. L'opposition " totale" du partisan radical d'une philosophie comme science rigoureuse à toute philosophie purement idéologique en général (en 1910-1911), a fait place à une reconnaissance, par celui qui est devenu le fondateur d'une philosophie comme science rigoureuse, des valeurs et du rôle historiques de cette idéologie par-

(77) Ceux aussi - phénoménologues ou interprètes et critiques de Husserl qui prétendent « critiquer » et « rejeter » la réduction phénoménologique, ne critiquent et ne rejettent, très souvent, que cette thèse idéaliste qu'ils substituent à elle. Ils ne reconnaissent pas, dans ce cas, qu'une critique de la méthode réductive constitue une tâche autonome et que, au surplus, dans la situation historique créée par Husserl, une critique préalable de la réduction elle-même est indispensable si l'on veut entrer dans une critique de l'idéalisme.

(28) Voir note 62. 
ticulière qui constituait une anticipation "intuitive " de ce qu' établira, grâce à la phénoménologie husserlienne, une philosophie scientifique. Le titre même d'un article publié par Husserl en 1923: Die Idee einer philosophischen Kultur, en joignant les deux mots de culture et de philosophique, manifeste une sorte de réconciliation entre l'idée d'une philosophie, entendue ici comme science rigoureuse, et les exigences d'ordre pratique et idéologique de la culture de notre époque ${ }^{(79)}$. Cette idée se trouve réaffirmée dans un passage final de cet autre article, rédigé en 1924, sur Kant und die Idee der Transzendentalphilosophie, où il est dit, au sujet de la philosophie transcendantale de Husserl lui-même: " un art fort inutile, elle ne sert pas les grands et les maîtres de ce monde, les politiciens, les ingénieurs, les industriels ... [Mais] peut-être remplit-elle, elle aussi, les fonctions théoriques d'une praxis, et peut-être d'une praxis précisément qui correspond aux intérêts ultimes et suprêmes de l'humanité " (80).

Pourtant, faut-il le répéter, il n'est pas question pour Husserl d'abandonner pour autant la moindre des exigences d'une philosophie véritablement scientifique ${ }^{(81)}$. Au contraire, il résumera à cette même époque, dans la partie finale d'une Kritische Ideengeschichte qui fait partie du cours sur l'Erste Philosophie dont nous parlions déjà, l'essentiel de ses critiques touchant le caractère d'idéologie de l'idéalisme (et du rationalisme) classique.

(i9) T. VII, pp. 203-207, ainsi que pp. 8-10 et 11-17.

(80) T. VII, p. 283.

(81) Cet idéal d'une philosophie comme science rigoureuse, Husserl ne l'abandonnera jamais. Il est vrai que de nos jours, on s'obstine à citer et à réciter ce fragment d'un texte husserlien de 1935 que voici: . La philosophie comme science, science sérieuse, rigoureuse, apodictiquement rigoureuse - le rêve en est fini...n. Cependant, lues dans leur contexte, ces paroles révèlent une signification contraire à celle qu'on leur attribue d'habitude. En effet, Husserl poursuit quelques lignes plus loin: "Telle est la conviction générale qui domine à notre époque. L'humanité européenne se trouve submergée par un torrent, sans cesse croissant, ... d’une philosophie qui renonce à toute aspiration scientifique s. Ce ne serait pas en de pareils termes qu'un philosophe confesserait son abandon d'un idéal qu'il aurait poursuivi pendant toute sa vie. Par ailleurs, ce contexte dont on a tort de séparer l'expression de « rêve qui s'est évanoui » est facilement accessible depuis quatre ans (t. VI, p. 508, dans Beilage XXVIII). Notons encore que Quentin LAUER, dans son introduction a la La philosophie comme science rigoureuse (p. 7; voir note 13), tout en s'opposant à l'interprétation ordinaire du mot, en propose une autre qui nous semble insuffisante. 
Le mot-clé des critiques exprimées par Husserl à l'égard de l'idéalisme allemand $\mathrm{du} \mathrm{XIX}^{\mathrm{e}}$ siècle ${ }^{\left({ }^{(82)}\right.}$ sera celui de reconstruction, auquel il substitue parfois des expressions telles que procédé régressif, méthode reconstructive ou simplement constructive ${ }^{(83)}$. Selon toute probabilité. Husserl a repris cette notion de reconstruction, tout en lui donnant un sens critique, à Paul Natorp ${ }^{\text {(84) }}$. Chez ce dernier, en effet, ce terme désigne la méthode par excellence d'une philosophie transcendantale en général.

Pour Husserl, Natorp fut le dernier représentant authentique de l'idéalisme classique et celui dans la pensée duquel cet idéalisme finit par prendre conscience, d'une manière explicite, de son rôle historique véritable en tant qu'idéologie; c'est que, précisément. Natorp reconnut que la méthode caractéristique de l'idéalisme classique était celle d'une reconstruction. Rappelons ici, d'autre part, que Husserl, dans sa lettre de 1918 déjà citée, semble heureux de pouvoir reconnaître, non seulement à un ouvrage particulier mais à l'œuvre de Natorp en général, " une valeur inestimable ... comme expression d'une idéologie " ${ }^{(85)}$. Comment, par ailleurs, ces paroles

(22) Ces critiques, notons-le, sont adressées par Husserl, non seulement aux philosophies de l'idéalisme allemand classique, mais aussi à celles du rationalisme des temps modernes - voire du moyen âge et de lantiquité - en général. Au début de son exposé, Husserl nomme expressément Descartes, Spinoza, Leibniz, Kant et Hegel ( $t$. VII, p. 182); plus loin, il mentionne les a écoles néokantistes * (ib., p. 194). A titre d'exemples pour une philosophie constructive, Husserl ne cite, explicitement, que celle de Kant et, moins souvent et moins directement, celle de Leibniz. Toutefois, il ne fait aucun doute que, dans l'esprit de Husserl, ses critiques concernent également, et même en premier lieu, les œuvres de Fichte, de Schelling et de Hegel.

(83) Il va sans dire qu'on ne devra point entendre ici les mots "construction ", " constructif, , " reconstruction », "régression », etc., dans le sens qu'ils ont d'ordinaire en frança is.

(81) Cf., par exemple, t. VII, pp. 189, 194, 370, 373 et 375 . Il est vrai que, dans tous ces passages, Husserl évite de mentionner expressément le nom de Natorp. Une analyse de la correspondance philosophique entre Husserl et Natorp, conservée aux Archives-Husserl à Louvain, apporterait des précisions intéressantes. Nous ne pouvons cependant remplir ici notre promesse, faite en 1955 déjà, d'examiner de plus près les relations entre ces deux philosophes (cf. notre introduction au t. VII, pp. XVIII-XX). Nous devons nous contenter des remarques générales qui vont suivre.

(88) Voir plus haut. Nous soulignons. 
husserliennes n'auront-elles pas rappelé à Natorp ce passage de Philosophie als strenge Wissenschaft où Husserl concluait ainsi : "Il n'y a qu'une solution: à savoir que la philosophie idéologique elle-même abandonne, avec une parfaite bonne foi, la prétention à être science et, par conséquent, qu'elle cesse en même temps ce qui est certainement contre son intention pure - de troubler les esprits et d'empêcher le progrès de la philosophie scientifique. Que sa fin idéale reste donc purement une idéologie, idéologie qui, par son essence, n'est pas, précisément, science " ${ }^{(88)}$. Nous avons vu plus haut que cette séparation nette de l'idéologie et de la science philosophique, solution idéale envisagée par Husserl dans Philosophie als strenge Wissenschaft, ne tient pas compte de la situation historique et des rapports dialectiques entre l'une et l'autre. Toutefois, cette opposition elle-même est réelle. Ainsi, nous pouvons retrouver cette même idée encore dans un texte husserlien de 1924 qui contient des remarques critiques contre la philosophie de Kant et du néokantisme. Explicitement, le passage que nous allons citer se réfère à Kant lui-même. En réalité, Husserl nous semble penser ici, une fois de plus, à Natorp: "Toutes ces critiques seraient erronées s'il ne s'était assigné pour but, en personnalité digne [de cette tâche], que de former - pour lui-même, mais aussi, par là, pour toute son époque qu'il représentait dignement - une idéologie universelle et une sagesse du monde, capables de nous guider d'une manière pratique et éthique et de nous rendre une position adéquate vis-à-vis de Dieu, du monde et du prochain ; [s'il en était ainsi,] nous n'aurions rien à objecter [à sa philosophie]" ( ${ }^{87)}$.

D'autre part, ces paroles nous montrent que, lorsque Husserl aborde le problème de la pensée reconstructive en décrivant une tendance de la philosophie moderne appelée par lui la " métaphysique de la conciliation ", il entend découvrir les racines historiques de ce style de pensée dans l'ordre de l'idéologie.

En effet, dit Husserl, " déjà dans l'école cartésienne nous pouvons constater une tendance à former une métaphysique qui aura pour motif de réconcilier une interprétation du monde déterminée par l'explication causale avec une autre dont le point de vue est théologique " ${ }^{(89)}$. "Métaphysiquement, la philosophie cartésienne...

(86) Logos, I, p. 338.

(87) T. VII, p. 375 (Beilage XVIII). Nous soulignons.

(83) Cette opposition établie par Husserl entre une pensée théologique, d'une 
semblait pousser vers une conception causaliste du monde qui ne pouvait satisfaire aux exigences de la religion et aux besoins d'ordre éthique liés à celles-ci. Ainsi, et déjà dans l’occasionalisme, ... des tontatives prennent naissance en vue de construire une métaphysique à partir de postulats d'un ordre religieux et éthique " ${ }^{(80)}$. "L'évolution ultérieure se trouve essentiellement dominée par un besoin qu'éprouve la philosophie de réconcilier une conception causaliste et naturaliste du monde avec une conception finaliste et spiritualiste, et la nécessité mathématico-mécanique avec la liberté humaine et divine. Cette tendance conciliatrice imprimera à ces tentatives métaphysiques un caractère méthodologique tout particulier ... " (80). "Lorsque, au XIX siècle, et à partir d'une interprétation déterminée de la critique kantienne de la raison, il est question d'une 'méthode transcendantale', alors on entend par là, en opposition avec ce que nous entendons par cette expression [si nous désignons ainsi la méthode de la phénoménologie], une méthode régressive et constructive dans un sens bien déterminé, méthode qui aura pour tâche de rechercher les 'conditions de possibilité' d'une connaissance objectivement valable ... Dès ses commencements, la métaphysique de la conciliation est reconstructive dans un sens semblable; et il me semble que c'est dans cette métaphysique reconstructive qu'il faut chercher l'origine historique primitive du fait que la reconstruction assumera un rôle tellement important dans l'épistémologie [préphénoménologique] " ${ }^{(01)}$.

Husserl attribue donc une signification et une importance bien plus larges à l'idée et aux procédés de reconstruction que ne leur accordait Natorp dans le cadre restreint de l'épistémologie. Quant à la " métaphysique de la conciliation", elle n'en offre pas seulement un exemple important, mais elle constitue l'origine historique et idéologique du rôle des procédés de reconstruction dans l'idéalisme du XIX siècle. Il serait pourtant erroné de croire que, pour Husserl, ce rôle de la reconstruction dans l'idéalisme allemand, de

part, et une explication purement causale du monde, d'autre part, se retrouve fréquemment dans son ceuvre et dans les inédits; cf., par exemple, t. III, pp. 138 139. Pour Husserl, la possibilité d'une théologie philosophique (et, plus généralement, d'une métaphysique telle que cette discipline est conçue par lui) est étroitement liće à l'idée d'une téléologie universelle.

(80) T. VII, pp. 188-189.

(00) Ib., p. 189.

(01) Ib., pp. 189-190. 
Kant à Natorp, s'explique uniquement par cette situation idéologique particulière qui, selon lui, est celle du début des temps modernes. Certes, l'origine historique de la philosophie d'une époque déterminée doit se trouver dans une situation historiquement concrète. Cependant, dans l'esprit de Husserl, la situation idéologique concrète du début des temps modernes qu'il décrit n'est sans doute qu'une expression particulière d'une " situation " plus générale de l'humanité et qui durera tant qu'une philosophie comme science rigoureuse n'aura fait son début. Il $s^{\prime}$ agit, en effet, de la situation historique d'une civilisation qui est fondée, au point de vue spirituel, sur une simple idéologie. Nous savons qu'à toute "culture idéologique ", Husserl oppose "l'idée d'une culture philosophique " dont parle le titre d'un article de cette époque, auquel nous faisions allusion plus haut ${ }^{(92)}$. Historiquement, les procédés de reconstruction sont essentiels à l'idéologie en général et en tant que telle. En ce sens, de tels procédés sont même historiquement nécessaires afin de permettre la construction d'une idéologie qui doit précéder, anticiper et rendre possible par là la mise en chantier d'une philosophie scientifique; celle-ci seulement pourra enfin abandonner ces procédés.

Cette relation nécessaire, entre la construction régressive et anticipative, d'une part, et un procédé " progressif " et véritablement scientifique, d'autre part, Husserl la décrit, dans notre contexte, à l'exemple de la situation particulière et individuelle de tout chercheur dans le domaine de la science: "En un certain sens, tout chercheur scientifique procède, évidemment, d'une manière constructive et régressive: il le fait au niveau [où il conçoit] l'idée inventrice. Toute invention présuppose une anticipation; l'on ne peut rien chercher et l'on ne peut commencer un travail productif sans posséder d'avance une idée directrice de ce qu'il faudra chercher ou de ce qu'il faudra produire. L'inventeur tentera à tracer d'avance, dans son imagination, les chemins possibles qui pourraient l'amener, par le moyen de vérités déjà établies et qui en formeront les jalons, au résultat anticipé. Tout cela. cependant, ne peut dépasser le stade du simple projet et le niveau d'une vraisemblance provisoire et doit être suivi d'un travail réellement et effectivement réalisateur ; celui-ci procédera, partant de fondements fermement 
établis et passant ensuite à ce qui peut être fondé sur eux, d'une manière progressive " ${ }^{(03)}$.

Le passage tout entier peut être transposé, sans difficulté, à la relation plus générale qui existe, historiquement, entre l'idéalisme tel qu'il se trouve anticipé d'abord sous forme d'idéologie et l'idéalisme tel qu'il sera établi, d'une manière " progressive " et véritablement scientifique, par le " travail réellement et effectivement réalisateur " de la phénoménologie de Husserl.

Tentons enfin de préciser ce que signifie la notion de reconstruction dont Husserl se sert pour désigner la structure méthodologique fondamentale de toute idéologie en général et, en particulier, de l'idéalisme allemand du $\mathrm{XIX}^{\ominus}$ siècle, pourtant essentiellement " vrai " dans le contenu de son enseignement ${ }^{\text {(94). }}$.

Est reconstructive, selon Husserl, toute pensée qui $s^{\prime}$ efforce de reconstruire, "logiquement ", et à partir d'un ou de plusieurs principes qui, à cette fin, sont posés d'avance, les données prétendues du monde réel et du monde idéal, ces données elles-mêmes étant admises d'avance comme telles; et cela de telle manière que la "réussite " de la reconstruction devra " prouver " ou légitimer les principes qui auront servi de base à cette opération. - Explicitons cette structure en en relevant, un à un, les moments essentiels.

Le principe - ou le système de principes - d'une philosophie idéologique relève originairement $d$ 'une anticipation "intuitive " ou d'un choix ; il est un pro-jet ou une pro-jection ; on peut l'appeler

(08) T. VII, p. 191.

(04) A raison des rapports intimes qui relient les idéologies au caractère historique et temporel de la vie pratique des hommes en général - rapports esquissés par Husserl dans l'article Philosophie als strenge Wissenschaft -, cette structure de "reconstruction" formera, en dernière analyse, un moment constitutif du « monde de la vie» en général. Bien qu'exprimée dans une terminologie différente, cette idée joue un rôle fondamental dans la théorie husserlienne du a monde de la vie ,, théorie développée dans Die Krisis der europäischen Wissenschaften und die transzendentale Phänomenologie, de 1936 (t. VI). Ce dernier écrit a pour thème principal le problème de l'a antagonisme spécifique qui oppose un engagement dans la dimension [d'une philosophie scientifique] à toutes les activités qui répondent aux intérêts constitutifs de la vie naturelle et normale des hommes au monde , (t. VI, p. (21). Cet antagonisme, Husserl l'a ressenti et décrit dans Philosophie als strenge Wissenschaft déjà lorsqu'il y oppose l'idée d'une philosophie scientifique qui réclame une époché universelle et la « vie " qui exige toujours des "prises de position »; voir plus haut et note 68. 
aussi un "pari " (95), fait sur la possibilité de reconstruire, à partir de lui, les "données" du monde réel et du monde idéal (96). Quant à la méthode elle-même. son aspect est double. D'une part, elle est une "déduction" des "données" réelles et idéales à partir du principe ou des principes du "pari ". Cette "déduction" ellemême peut prendre des formes diverses ; elle peut relever, méthodologiquement, d'une simple logique formelle ou d'une logique transcendantale (déductions au sens kantien ou fichtéen) ou encore d'une logique dialectique (au sens hégélien, par exemple). D'autre part, cette méthode doit servir aussi, sinon en premier lieu, à légitimer le principe ou le système de principes qui sont posés d'avance ; sous ce dernier rapport. elle se présente comme une méthode de "vérification " (97). Conformément à ce double aspect de la méthode

(05) En effet, les interprétations husserliennes peuvent faire penser au fameux pari de Pascal. Dans le cadre de l'interprétation, proposée par Husserl, de la situation idéologique de l'époque cartésienne, la pensée de Pascal devait appartenir à la "métaphysique de la conciliation " des temps modernes. De toute façon, « une interprétation qui fait du pari le centre de la pensée pascalienne *, comme celle fournie par Lucien Goldmann dans Le dieu caché. Etudes sur la vision tragique dans les Pensées de Pascal et dans le théâtre de Racine (Paris, 1955. p. 322) peut sembler confirmer les vues historiques husserliennes que nous analysons ici. Par ailleurs, L. Goldmann met aussi en parallèle " la pensée centrée sur le 'pari' ' de Pascal avec la philosophie de Kant. L'arrangement des Pensées dû à Léon Brunschvicg, qu'on pourrait appeler le Natorp français, semble favoriser les thèses de Goldmann; pour a la méthode dans la philosophie de l'esprit » de Brunschvicg, voir par exemple L'idéalisme contemporain (Paris, 1905), pp. 73-97. Mais n'insistons pas. Surtout qu'il est certain que Goldmann ne souscrit nullement aux principes de Brunschvicg. Toutefois, Goldmann attribue une valeur positive à l'idée du "pari » en général et se déclare ainsi partisan d'une philosophie "idéologique" au sens husserlien.

(0o) Il est évidemment possible que ces principes projetés ne soient point expressément énoncés dès le début du travail philosophique qui vise la reconstruction des a données \$.

(97) Ce moment, par exemple (cf. note 58), peut trouver une belle illustration par des critiques tout analogues qu'adresse le jeune Marx à la philosophie hégélienne de l'Etat. Chez Hegel, selon Marx, a l'essence des déterminations de l'Etat ne consiste pas en ceci précisément qu'elles sont des déterminations de l'Etat, mais en ce qu'elles peuvent être considérées, dans leur forme la plus abstraite. comme des déterminations logico-métaphysiques. Ce qui intéresse véritablement [Hegel, dans ses Grundlinien der Philosophie des Rechts, 1821], ce n'est pas la philosophie du droit mais c'est la logique. Le travail philosophique ne consiste pas à donner corps à la pensée sous la forme de déterminations politiques, mais a subtiliser les déterminations politiques données qui sont réduites à des pensées abstraites. Le moment philosophique ne consiste pas dans la logique de l'objet, 
reconstructive, le résultat de son application sera également double. D'une part, l'aboutissement des déductions à une reconstruction du monde réel et du monde idéal doit " prouver " (" vérifier ") le principe initial. Il le doit, d'autre part, en re-présentant, précisément, les "données " qu'on aura réussi à reconstruire.

Deux choses paraissent ici immédiatement. D'une part, il semble évident que cette reconstruction du monde ne pourra jamais être complète et exhaustive : on se limitera, inévitablement, à en déduire l'“ essentiel ». Or, c'est là une conséquence grave du choix initial des principes: le procédé tout entier est manifestement trompeur en ce que, sournoisement, il part d'une distinction "apriorique " implicite et arbitraire de ce qui sera "essentiel" et de ce qui ne le sera pas; l'un destiné à être "reconstruit ", l'autre à être simplement éliminé. En outre, il est clair que la déduction reconstructive ne laissera point les " données " à reconstruire sans les soumettre à des modifications importantes, selon la lumière et selon la place auxquelles ces "données " réapparaîtront au cours de leur reconstruction ${ }^{(8)) \cdot(89)}$.

mais dans l'objet de la logique. Ce n'est pas la logique qui sert à démontrer l'Etat, mais c'est l'Etat qui sert à démontrer la logique "(MarX-Encels, Werke, l. c., t. I, p. 216).

("*) Ici encore, un passage de la critique marxiste de la philosophie hégélienne de l'Etat peut servir d'illustration. Commentant le $\$ 272$ de la Philosophie des Rechts de Hegel, Marx fait remarquer: «La constitution [d'un Etat] est donc raisonnable pour autant que ses moments peuvent être résolus en des moments abstraits de la logique. La réalité de l'Etat se détermine et se distingue [en luimêmel, non selon sa nature spécifique, propre à lui, mais selon la nature du concept qui constitue le mobile mystifé de la pensée abstraite. La raison de la constitution réside donc dans une logique abstraite, et non dans le concept de l'Etat. Au lieu d'un concept de constitution, on nous offre la constitution du concept. Ce n'est pas la pensée qui se conformera à la nature de l'Etat, mais $c^{\prime}$ est au contraire l'Etat qui s'accommodera à une pensée déjà toute faite » $l$. c., t. 1, pp. 217-218).

(99) On remarquera qu'une structure de a reconstruction * semble aussi caractéristique de toute méthode $d^{\prime}$ «interprétation » philologique, historique ou philosophique. On interprète, par exemple, un texte philosophique en partant d'une « idée " générale - explicite ou non dans le texte - qu'on considère comme sous-jacente au développement de ce texte; ce présupposé. on cherchera ensuite à l'établir en démontrant que le contenu a essentiel du texte peut se dériver de l'idée imaginée. Si telle est la structure de toute interprétation en tant que telle, on reconnaît aisément que l'idée même d'interprétation implique des difficultés analogues à celles qui sont inhérentes à toute méthode de reconstruction au sens défini par Husserl. On sait, par ailleurs, que cette problématique touchant l'idée 
Par ces dernières remarques, relatives au sort réservé aux " données" dans les reconstructions opérées par les idéologies, nous venons de nous approcher du point central des critiques husserliennes à l'égard de celles-ci. Husserl, en effet, s'opposera autant à la manière dont on conçoit ici les principes et dont on entend les " prouver" qu'à la méthode déductive elle-même - quel que soit le sens particulier de la déduction - qui servira à reconstruire les " données" et à " vérifier " les principes. Mais il critiquera surtout le " dogmatisme " qui préside, en de tels procédés, à la conception des " données " elles-mêmes. Ce que Husserl reprochera en premier lieu et à titre de "dogmatisme " à la pensée reconstructive, c'est en un certain sens que nous allons préciser - qu'elle prend pour point de départ, en dernière analyse, des prétendues "données", les prétendues " choses elles-mêmes", les prétendus " phénomènes".

En effet. le procédé en question ne part de prétendus " phénomènes ", " réels " et "idéaux ", dont la connaissance et la certitude sont supposées évidentes et qui ne seront jamais remis en question, que pour s'en départir aussitôt et n'y revenir que d'une manière "régressive ", à partir alors des principes projetés. Etant donné que ces principes ne seront légitimés que par la réussite d'une reconstruction, sur leur fondement, du monde supposé " phénoménal", donné réellement ou dans une objectivité idéale, les reconstructions idéologiques présupposent toujours ce dont il faudra établir, en philosophie, les fondements.

La philosophie idéologique " a devant elle un monde intuitif et des sciences objectives tout faites, déterminant des vérités pour elle, et elle a ses convictions religieuses et morales; ses réflexions ne concerneront que tout cela qui lui sert de point de départ: [elle tâche d'imaginer] comment il faudra repenser et interpréter ces réalités afin de satisfaire aux exigences de la science, de la religion et de la morale " ${ }^{(100)}$. Chez Kant, par exemple, "le procédé d'une méthode régressive joue un rôle de premier plan: comment [se

d'interprétation a fait l'objet, dans le cadre de recherches relatives à l'horizon le plus général où elle s'inscrit, d'une analyse pénétrante qu'on doit a Martin HEIDEGGER, dans Sein und Zeit (1927). Implicitement, le problème husserlien de la a reconstruction $s^{\prime} y$ trouve renouvelé.

(100) T. VII, p. 192. 
demande-t-il] sont possibles les mathématiques pures, les sciences de la nature pures, etc. ; comment faut-il concevoir la sensibilité afin de rendre possibles les jugements d'une géométrie pure ; comment les données diverses de l'intuition sensible doivent-elles trouver une unité synthétique afin de rendre possible une science rigoureuse de la nature, c'est-à-dire une détermination des objets de l'expérience sous la forme de vérités valables en elles-mêmes ? Kant luimême exige et effectue des 'déductions', à savoir les déductions, appelées par lui métaphysiques et transcendantales, des formes de l'intuition et des catégories; il déduit de même le schématisme, la validité nécessaire des principes de l'entendement pur, etc. Il est vrai que tout n'est pas simplement déduit et que ces déductions ont un sens différent de celui des déductions ordinaires. Et pourtant, il s'agit là d'un procédé constructif de la pensée, qui n'est rejointe qu'ensuite par l'intuition; il ne s'agit point d'un rendre compréhensible ... qui commence par en bas et qui s'élève ensuite [à des niveaux supérieurs], progressant de manière intuitive et $s^{\prime}$ en tenant exclusivement à ce qui peut [effectivement] être rendu présent " (101).

"Or, pour celui qui entreprend des recherches épistémologiques, le monde et la science ne sont point simplement des faits; ils ne sont pas des réalités simplement données dont il n'aurait qu'à prendre connaissance ; la vérité scientifique n'est pas une vérité simplement valable qu'il n'y aurait qu'à 'découvrir' dans une activité de son esprit, qu'il n'y aurait qu'à développer théoriquement, dont il n'y aurait qu'à faire ressortir les rapports intrinsèques nécessaires; mais tout cela ne sont que de 'faits visés et intuitionnés' par la subjectivité connaissance, posés dans son expérience immanente et dans ses productions théoriques, dans ses signi-fications ${ }^{(102)}$ immanentes et ses affections passives, dans ses réalisations actives et perpétuellement créatrices de sens, et, en particulier, dans ses légitimations et justifications 'évidentes' dans un sens privilégié " (103).

"Il n'est point suffisant de disposer de connaissances toutes faites comme de phénomènes et d'adresser à celle-là des questions touchant leur sens et leur validité objective; il faut s'aviser de la nécessité d'étudier, avant toute autre chose, ces phénomènes eux-

(101) Ib., pp. 197-198.

(102) Sinngebungen, \& donations de sens ".

(103) T. VII, pp. $371-372$ (Beilage XVIII, texte de 1924). 
mêmes en tant qu'ils sont des phénomènes et les soumettre, en tant qu'ils sont des phénomènes de l'intentionalité, à une explicitation intentionnelle " ${ }^{(104)}$.

Ce qui fait défaut à la pensée de l'idéalisme classique, défaut qui la pousse vers la construction et des procédés de reconstruction et qui la maintient au niveau d'une pure idéologie, c'est précisément une "science fondamentale ": une phénoménologie pure (105).

Cette phénoménologie, au sens husserlien, ne partira pas de phénomènes admis et présupposés d'avance comme étant généralement connus et universellement disponibles, pour s'enquérir simplement de ce qu'on pourra affirmer au sujet d'eux; et non plus pour rechercher, partant de là, une " définition " adéquate et convenable de la notion de " phénomène ". Au contraire. cette phénoménologie orientera ses recherches, et immédiatement, dans la direction des phénomènes eux-mêmes, et cela en ce sens qu'elle sera conduite par cette unique question: Qu'est-ce qu'on peut et qu'est-ce qu'on doit, en toute rigueur, considérer comme étant un phénomène véritable ? En effet, s'il est vrai que la philosophie aura pour tâche d'établir le fondement réel et la vraie raison de ce qui s'offre à nous en tant que " phénomène ", il est vrai aussi que la philosophie ellemême doit se fonder sur une phénoménologie préalable, systématique et complète; qu'il lui faut d'abord et avant toute autre chose rechercher, relever et décrire ce que sont les "phénomènes " dont elle devra établir, par la suite ${ }^{(106)}$, le fondement et la raison. Toute philosophie qui ne se fonde pas, en philosophie phénoménologique. sur une phénoménologie explicite et préalable, partira d'une " phénoménologie " implicite et dogmatique, présupposant " naturellement évident " ce sur quoi elle aura à se prononcer.

(104) T. VII, p. 195; souligné par nous.

(105) On sait que ce titre de " phénoménologie » a été inventé par J. H. Lambert et repris de lui, à quelques rares occasions, par Kant et Herder. Moins connu est l'usage important que, avant Hegel, Fichte a fait de ce terme (d'abord dans la Wissenschaftslehre de 1804, puis dans la Sittenlehre de 1812). Nous avons reproduit plus haut une note de Husserl sur la phénoménologie hégélienne (voir note 48). A l'époque de Husserl, par ailleurs, le terme de " phénoménologie » était employé par beaucoup d'auteurs et en des sens très divers.

(108) a Par la suite : en réalité, Husserl devait bientôt se rendre compte du fait qu'il ne pourra être question d'élaborer d'abord une phénoménologie simplement descriptive et d'établir ensuite seulement le fondement ou la raison des phénomènes qu'aura mis au jour une phénoménologie pure au préalable. Etablir les phénomènes purs veut dire établir leur logos, au sens prégnant de ce mot. 
Une telle philosophie, dogmatique, jugera de son objet sans en avoir examiné d'abord la signification et donc sans avoir véritablement pris connaissance de ce dont elle jugera. Une telle philosophie manque donc de la rigueur spécifique qu'il faut exiger d'un procédé véritablement scientifique. Elle n'est qu'idéologie. Au lieu de s'adonner, d'abord et avant toute autre chose, à un examen consciencieux et patient de ce qui se trouve soumis à son jugement, elle espère, pressée éventuellement par l'urgence du temps, pouvoir se dispenser du travail dur et ingrat qu'implique un tel examen, en inventant un principe - ou un ensemble de principes - à partir duquel - ou desquels - elle pourra, du coup, tout embrasser et tout expliquer.

Par contre, les exigences de rigueur pour celui qui veut établir une philosophie comme science rigoureuse lui imposent, avant de lui permettre de juger sur quoi que ce soit, une suspension universelle du jugement, c'est-à-dire une suspension de tous les jugements réels ou possibles qui exprimeront ou qui impliqueront, de quelle façon que ce soit, des pré-jugés. De la manière la plus scrupuleuse, il devra s'abstenir de juger avant d'avoir analysé jusqu'au fond ce sur quoi il aura à se prononcer. La tâche d'une phénoménologie pure implique l'exigence rigoureuse d'une époché universelle et absolument radicale.

"Ce qui est nécessaire avant toute autre chose ", note Husserl en résumant ses critiques dirigées contre le rationalisme et l'idéalisme classiques. " c'est de fixer méthodiquement, d'une façon générale et, pour ainsi dire, pédante [la maxime que voici] : toute connaissance, commençant par la connaissance qui relève de l'expérience la plus simple et jusqu'à toutes [les connaissances obtenues par] les sciences, doivent être traitées comme épistémologiquement problématiques ; et conformément à la signification de cette problématique, il faut d'emblée, au lieu de considérer toutes ces connaissances comme valables et d'en faire simplement usage, les concevoir (et aussi bien les objets qu'elles visent que les vérités qui, prétendument, déterminent ces objets) exclusivement telles qu'elles s'offrent à nous en tant que phénomènes " (107).

Husserl continue immédiatement ainsi : "Or, phénomène, toute connaissance l'est pour moi dans une subjectivité transcendantale : ainsi donc, l'exigence première d'une méthode pure et authentique 
consiste à ne rien poser en dehors de cette donnée qui, en effet, est en soi la première de toutes: la subjectivité transcendantale 'absolument' évidente " (108). En effet, on le sait, l'exigence d'une époché universelle, et donc l'idée même d'une phénoménologie pure. conduit, selon Husserl, à celle d'une réduction phénoménologique et transcendantale.

\section{$\mathbf{X}$}

Nous ne pouvons pas et nous ne devons pas analyser ici les rapports qui s'établissent, dans l'esprit de Husserl, entre l'exigence d'une époché universelle d'une part, exigence compréhensible, d'une manière générale, à partir de l'idée directrice d'une philosophie rigoureusement scientifique, et, d'autre part, la nécessité d'une méthode de réduction phénoménologique. Il doit nous suffire de préciser que c'est la mise en ouvre d'une méthode de réduction phénoménologique, dans une philosophie comme science rigoureuse, qui est opposée par Husserl, en dernière analyse et le plus radicalement, à l'application d'une méthode de reconstruction dans une philosophie qui demeure une simple idéologie. En effet, Husserl reproche à la philosophie du rationalisme et de l'idéalisme classiques en premier lieu qu" "elle ne voit point la méthode exigée pour une science transcendantale authentique " (109). Dans l'article sur Kant und die Idee der Transzendentalphilosophie, il souligne spécialement " que [même] une position transcendantale ... ne peut

(10:) Ib., p. 195. - Reproduisons ici un troisième passage essentiel des critiques adressées par le jeune Marx à la philosophie de Hegel. Reconnaîtra-t-on, ici encore, non seulement une assonance terminologique peut-être fallacieuse, mais une análogie réelle avec les observations husserliennes ?

Marx note: a Hegel donne une indépendance autonome aux prédicats, aux objets, mais il le fait en les séparant de leurs indépendance et autonomie réelles. c’est-à-dire, de leur sujet. Par la suite, le sujet réel apparaîtra comme un résultat, tandis qu'il fallait partir du sujet réel et en observer l'objectivation. [Hegel] fait donc d'une substance mystique un sujet réel, et par conséquent le [véritable] sujet réel apparaît comme autre chose, à savoir comme n'étant qu'un moment de cette substance mystique. Parce que Hegel, au lieu de partir de l'ens réel (de l'hypokeimenon, du sujet), part des prédicats, de la détermination universelle, et parce qu'il lui faut pourtant un suppôt à cette détermination, c'est l'idée mystique qui devient ce suppôt. Voilà le dualisme hégélien qui ne considère ni l'universel comme l'essence réelle du fini réel, c’est-d̀-dire de l'existant déterminé, ni cet ens réel comme le sujet véritable de l'infini ( $(l$. c., t. I, pp. 224-225).

(109) T. VII, p. 197. 
encore passer pour une position transcendantale authentiquement scientifique et philosophique, tant qu'une réflexion méthodologigue appropriée n'aura pas éclairé, plus profondément, la signification et la légitimité de cette exigence de pureté qui est en question ici, qu'une méthode scientifiquement légitimée n'aura assuré l'établissement d'une expérience transcendantale et, par là, ouvert le chemin vers la fondation originelle d'une philosophie transcendantale.comme science rigoureuse.

"Cette exigence, c'est la nouvelle phénoménologie qui lui a suff, sous le titre de 'réduction phénoménologique' "(110).

C'est donc cette méthode d'une réduction phénoménologique qui fondera la phénoménologie husserlienne, c'est elle qui permettra la constitution, sur le fondement d'une phénoménologie pure, d'une philosophie comme science rigoureuse, et c'est elle encore qui conduira Husserl à établir, sur des bases scientifiques, et non plus simplement idéologiques, un nouvel idéalisme. L'idéalisme phénoménologique de Husserl, en effet, se fonde sur la mise en ceuvre d'une méthode de réduction transcendantale. Mais, c'est aussi par le fait qu'il peut s'appuyer sur des résultats obtenus grâce à une méthode de réduction phénoménologique que cet idéalisme husserlien se distingue radicalement et essentiellement de l'idéalisme classique. Si donc il est vrai que, dans la pensée de Husserl, l'idéalisme se réalise (sous une forme scientifique, c'est-à-dire phénoménologique) tout en se dépassant (en dépassant ce qui le constituait en idéologie), cette réalisation et ce dépassement d'une idéologie idéaliste s'accomplissent sous la forme de la réduction phénoménologique. Pour cette idéologie idéaliste, la réduction phénoménologique aura donc la signification d'une $A$ ufhebung au sens hégélien (111).

Au fait, si les résultats de la philosophie phénoménologique husserlienne conduisent vers un rétablissement des résultats anticipés par l'idéalisme et par le rationalisme classiques des temps modernes, sa méthode - comme aussi, en un sens, ses problèmes fondamentaux - a ses racines historiques dans une autre tradition: celle de l'empirisme anglais. Dans les textes que nous analysons ici,

(130) T. VII, p. 255; cf. aussi, par exemple, t. VII, pp. 370, 371, 373, 382, 401, 404.

(111) Par ailleurs, comme l'idée hégélienne d'Aufhebung, l'idée husserlienne de réduction comporte trois moments: réduire veut dire défaire (supprimer), limiter (retenir en certaines limites) et ramener (à une position plus fondamentale ou " supérieure $»$ ). 
Husserl rappelle, au début de l'examen critique de la pensée rationaliste et idéaliste des temps modernes, que " ce fut la grande mission de l'empirisme, dans l'époque moderne, d'ouvrir le chemin à cette méthode du retour aux origines phénoménologiques de toute connaissance qui seule pouvait rendre possible la fondation d'une philosophie et d'appuyer l'exigence d'une philosophie radicalement intuitioniste " (112).

C'est pourquoi aussi, explique Husserl, il consacra la plus grande partie de son exposé historique et critique ${ }^{(113)}$ aux théories empiristes: "En elles, un nouveau type de méthode a fait son apparition avec Locke, et nos critiques de cette méthode avaient pour but de déceler, dans cette méthode de psychologie immanente, une tendance historique vers une méthode vraiment philosophique et absolument nécessaire, la méthode phénoménologique " ${ }^{(114)}$, c'està-dire celle de la réduction. En effet, après la découverte du cogito, due à Descartes, "Locke fut le premier à chercher un chemin, du cogito cartésien, vers une science de ce cogito, et le premier à exiger, au point de vue de la méthode,... gu'on réduise toute connaissance à ses sources originelles et intuitives dans la conscience, dans l'expérience immanente, et qu'on l'explicite à partir de ces sources originelles " (115). On doit à Berkeley, selon Husserl, "la première tentative systématique en vue de rendre théoriquement compréhensible la constitution du monde réel (du monde physique et du monde des animaux et des hommes) dans la subjectivité connaissante " (116). "Le Treatise de Hume ", enfin, " constitue le premier projet d'une phénoménologie pure, bien que celle-ci y prît la forme d'une phénoménologie empirique et sensualiste " ${ }^{1117 ;}$. Chez Hume aussi, apparaît pour la première fois dans l'histoire de la philosophie la notion de "réduction" dans son acception moderne, sous la forme de l'idée d'une "réduction de toutes les idées à des impressions " ${ }^{(118)}$.

(112) T. VII, p. 182

(in) Dans la Kritische Ideengeschichte de 1923, constituant la première partie du cours sur l'Erste Philosophie, t. VII.

(114) T. VII, p. 187.

(115) Ib., p. 144.

(118) Ib., p. 150.

(127) Ib., p. 157. - David HUME, A treatise on human nature, 1738-1740.

(118) Ib., p. 160. - Remarquons que toutefois, la notion de réduction (reductio) a une histoire bien plus ancienne dans la philosophie de l'Occident. Elle a surtout joué un rôle important dans le néoplatonisme latin. 
Peut-être objectera-t-on: Quelle que soit l'étendue des influences empiristes que, surtout dans la première phase de son évolution, Husserl ait subies, et des enseignements que, dans les périodes ultérieures encore, il ait tirés des recherches et des doctrines de Locke, de Berkeley et de Hume, il reste que ce fut en opposition radicale à cet empirisme, précisément, que Husserl fonda, dans les Logische Untersuchungen, ce qu'il appellera une "phénoménologie pure". $E_{11}$ outre, dans ce même ouvrage, Husserl s'appliquait à montrer que toute doctrine empiriste ou apparentée aboutit nécessairement, en dernière analyse, à un scepticisme qui exclut la possibilité même d'un véritable savoir scientifique. Comment alors retenir la thèse que cette méthode phénoménologique par laquelle Husserl entend réaliser l'idée d'une philosophie comme science rigoureuse soit, elle, d'inspiration essentiellement et originairement empiriste ?

En réalité, répondrons-nous, la conclusion la plus importante des Logische Untersuchungen fut exactement celle qu'exprime l'article Philosophie als strenge Wissenschaft en résumant une fois de plus les critiques husserliennes à l'égard de l'empirisme: "Nous pouvons peut-être dire que le psychologisme, en marche depuis le temps de Locke, n'était, en fait, qu'une forme voilée sous laquelle la seule tendance philosophique légitime - vers une fondation phénoménologique de la philosophie - était obligée de progresser. D'ailleurs, en tant que l'étude phénoménologique est une étude d'essences, et par là dans le vrai sens $a$ priori, elle tient compte en même temps de tous les motifs légitimes de l'apriorisme. De toute façon, notre critique aura mis en évidence que, reconnaître le naturalisme comme une philosophie erronée en principe, ne revient nullement à abandonner l'idée d'une philosophie rigoureusement scieniifique, d'une "philosophie d'en bas" " ${ }^{(110)}$. Ce texte est des plus instructifs ; il montre à l'évidence que, pour Husserl, la conception d'une philosophie comme science rigoureuse est d'abord liée aux tendances de cette " philosophie d'en bas " qu'est l'empirisme traditionnel: de sorte qu'il admet - en le contestant - que cette idée semble être remise en question dès qu'on s'attaque aux principes, aux méthodes et aux enseignements de cet empirisme. Au fait, Husserl n'entend s'opposer à l'empirisme traditionnel qu'afin d'en transformer le positivisme en un " positivisme " plus pur et entière-

(110) Logos, I, p. 322 . 
ment dépouillé de tout élément constructif ${ }^{(120)}$ : il s'agit, pour lui, " de substituer à ce négativisme sceptique (qui s'appelle positivisme) un positivisme vrai " (121). Tout en acclamant une philosophie et une psychologie qui ont pour devise: "Ce sont les choses ellesmêmes que nous devons interroger. Faisons retour à l'expérience, à l'intuition qui seule peut rendre à nos paroles un sens et un droit rationnel ". Husserl leur retourne cependant la question de savoir: "Très bien! Mais qu' est-ce donc que ces choses, et quelle est cette expérience à laquelle il faut faire retour ...? " (22). On sait que, à l'époque des Logische Untersuchungen, Husserl entend surtout reposer ainsi le problème d'une intuition "éidétique " et celui du statut des "idées", des objectivités idéales. En établissant un fondement phénoménologique qui permet une solution "positive " de ces derniers problèmes, il établit aussi, du coup, un " positivisme vrai " qui peut surmonter le scepticisme, inhérent à l'empirisme traditionnel.

ll est vrai qu" " au fond, l'empirisme continue la tradition du négativisme de la philosophie sceptique de l'antiquité, le rationalisme, lui, celle dont l'évolution, constructive dans un sens positif. se dirigeait vers une science vraie et entièrement parfaite et donc vers une philosophie authentique. Ce dernier continue la tradition du platonisme et du réalisme médiéval ; il est opposé à tout nominalisme qui veut mettre en doute les idées universelles et toute connaissance véritablement rationnelle; il est défenseur des vérités 'éternelles' " ${ }^{\text {(23). }}$. Cela veut dire, pour Husserl: c'est au rationalisme et à l'idéalisme classiques qu'appartient le mérite d'avoir défendu une foi dont l'humanité a et avait besoin. Mais, d'autre part, les empiristes " sceptiques " n'avaient pas tort absolument, en fin de compte, de ne point partager cette foi. En effet, l'idéal de l'idéologie idéaliste elle-même exige d'être appuyé, non par une simple foi, mais par la raison, par le savoir, et par la science: par une philosophie comme science rigoureuse. De cette dernière, qui ne sera mise sur pied que par la phénoménologie husserlienne, destinée à rétablir la vérité scientifique des doctrines idéalistes, l'empirisme, bien que "sceptique ", était plus près, en un sens, que

(120) Cf. Ideen (I), t. III, pp. 43 et 46 ; pp. 65 et 69 de la traduction française. (121) Logos, I, p. 340.

(122) Ib., p. 305.

(120) T. VII, pp. 182-183. 
l'idéalisme classique lui-même. "A cet égard ", dira Husserl, "le négativisme sceptique fut plus positif que le rationalisme qui progressait dans un travail positif rationnel ") (124).

La démarche husserlienne prit son départ de l'idée d'une " philosophie d'en bas ", idée dont elle ne se départira jamais complètement. Son idéal scientifique qui devint aussi son idéal philosophique était un idéal " positiviste ". Consciemment, Husserl s'opposait toujours à toute philosophie constructive ou reconstructive, "d'en haut". Jamais le but de cette démarche husserlienne n'a été d'appuyer ou d'établir une "idéologie " quelconque. Cependant, ses recherches l'amenèrent, à l'époque des Logische Untersuchungen, à une transformation radicale des conceptions du positivisme traditionnel dont il dénonça les conséquences sceptiques sans fondement. Il instaura une phénoménologie nouvelle à la place de la phénoménologie sensualiste et purement empirique de l'empirisme ancien. Et cette nouvelle phénoménologie l’amena, grâce à l'application rigoureuse d'une méthode de réduction, à reconnaître comme une vérité qui s'impose à une philosophie comme science rigoureuse, "d'en bas", une conception du monde qu'avait anticipée, sous forme de simple idéologie, l'idéalisme allemand classique du siècle précédent.

Nous ne nous proposons point ici d'entrer dans une discussion critique des vues husserliennes que nous venons d'exposer: nous avons même renoncé à examiner l'interprétation husserlienne de la philosophie de l'idéalisme classique en faisant retour aux textes des ceuvres issues de cette dernière philosophie elle-même. Cependant, nous n'estimons point, d'une manière générale, qu'une séparation absolument nette soit possible entre une analyse purement historique qui se limiterait à vouloir simplement comprendre une philosophie, d'une part, et un jugement critique sur cette philosophie, d'autre part, qui s'appuyerait sur cette analyse préalable. C'est que, seulement ce qui est vrai est aussi susceptible d'être vraiment compris, ou que c'est seulement dans la mesure où une philosophie est vraie, qu'elle est aussi réellement compréhensible. En le disant. avouons-le, nous exprimons tout de même déjà une première cri-

(126) Ib., p. 187. 
tique à l'adresse des conceptions husserliennes. Mais par là encore, nous admettons aussi une fois de plus n'avoir évidemment poussé la compréhension de la pensée husserlienne et de ses rapports à l'idéalisme classique, ainsi qu'au problème de l'idéalisme en général, que jusqu'à une certaine limite. Toutefois, à partir des résultats acquis dans la présente étude préliminaire, une analyse approfondie de la signification historique et philosophique de la pensée et de l'cuvre de Husserl pourrait s'amorcer, et elle aurait à poursuivre les questions suivantes:

L'idée d'une philosophie comme science rigoureuse exige-t-elle, comme science fondamentale, une phénoménologie ?

L'idée d'une phénoménologie conduit-elle nécessairement à l'application d'une méthode de réduction, au sens husserlien ?

L'application d'une méthode de réduction phénoménologique conduit-elle réellement à l'établissement d'un idéalisme philosophique absolument fondé ?

Qu'est-ce, en fin de compte, qu'un idéalisme philosophique, à supposer qu'il ne puisse se réaliser que par la mise en œuvre d'une méthode de réduction phénoménologique ?

Quelle est l'origine de l'idée d'une philosophie comme science rigoureuse?

Rudolf BOEHM

Louvain. 